\title{
Breathing on Chip: Biomechanical forces change airway epithelial cell biology in a human Airway Lung-Chip
}

Janna C. Nawroth ${ }^{1,2}$, Doris Roth ${ }^{2 \dagger}$, Annemarie van Schadewijk ${ }^{4}$, Abilash Ravi ${ }^{4}$, Tengku Ibrahim Maulana ${ }^{2 \dagger}$, Christiana N. Senger ${ }^{1}$, Sander van Riet ${ }^{4}$, Dennis K. Ninaber ${ }^{4}$, Amy L Ryan ${ }^{1,6}$, Pieter S. Hiemstra ${ }^{4}$, Anne M. van der Does ${ }^{4}$

${ }^{1}$ Hastings Center for Pulmonary Research, Division of Pulmonary, Critical Care and Sleep Medicine, Department of Medicine, University of Southern California, Los Angeles, CA, U.S.A.

${ }^{2}$ Emulate Inc., Boston, MA, U.S.A.

${ }^{4}$ Dept. of Pulmonology, Leiden University Medical Center, Leiden, The Netherlands

${ }^{6}$ Department of Stem Cells and Regenerative Medicine, University of Southern California, Los Angeles, CA, U.S.A

tCurrent address. DR: Vienna University of Technology, Faculty of Technical Chemistry, Institute of Applied Synthetic Chemistry and Institute of Chemical Technologies and Analytics, Vienna, Austria; TIM: Department of Biomedical Science, Faculty of Medicine, Eberhard Karls University of Tübingen, Tübingen, Germany.

\section{Corresponding author:}

Anne M. van der Does, PhD, Department of Pulmonology, Leiden University Medical Center, P.O. Box 9600, 2300 RC Leiden, The Netherlands

E-mail: a.van_der_does@lumc.nl

Author contributions: Conception and design: JN, DR, AD. Sample preparation: JN, DR, AS, TIM, CS, SR, $D K, A D$. Analysis and interpretation: JN, DR, AS, AR, PH, AD. Drafting the manuscript for important intellectual content: JN, AS, AR, JN, ALR, PH, AD. All authors have read the manuscript, provided input, and agree with its submission.

Key words: organs-on-chips; airway epithelium; differentiation; breathing; airflow; biomechanics 


\begin{abstract}
Human lung function is intricately linked to the mechanics of breathing; however, it remains unknown whether and how these mechanical cues shape human lung cellular biology. While respiration-related strains and fluid flows have been suggested to promote alveolar epithelial cell function, the study of such fundamental mechanisms in the conducting airway epithelium has been hindered by the lack of suitable in vitro airway models. Here, we developed a model of human bronchial airway epithelium using welldifferentiated primary cell cultures on a commercial Organs-on-Chips platform that enables the application of breathing-associated airflow and cyclic strain. It furthermore features optional endothelial cell co-culture to allow for crosstalk with the vascular compartment. Using this model, we evaluated the impact of airflow and physiological levels of cyclic strain on airway epithelial cell differentiation and function. Our findings suggest that breathing-associated mechanical stimulation changes epithelial composition, reduces secretion of IL-8, and downregulates gene expression of matrix metalloproteinase 9, fibronectin, and other extracellular matrix (ECM) factors. These results indicate that breathingassociated forces are important modulators of airway epithelial cell biology and that their fine-tuned application could generate models of specific epithelial phenotypes and pathologies.
\end{abstract}




\section{Introduction}

Breathing is an essential part of mammalian biology, and every breath generates dynamic cycles of airflow and tissue strain throughout the lungs ${ }^{1-3}$. Recent experimental and theoretical work has revealed a diverse mechanical landscape along the respiratory tree where tissue strain tends to increase as a function of branching generation, whereas airflow velocity reduces ${ }^{1,3}$. It is, however, largely unknown how these mechanical force and velocity gradients relate to the well-known variation in epithelial cell composition as a function of branching generation ${ }^{4,5}$. In the larger airways, the pseudostratified epithelium comprises of a variety of cells, including basal cells, ciliated cells, and mucosecretory cells (i.e., goblet and club cells) that promote microbial clearance from the lungs. Towards the small airways, the proportion of basal and goblet cells decreases as club cells become more abundant, until the alveolar epithelium is reached where gas exchange takes place. Whether mechanical cues play a role in establishing these differential cell populations is currently unknown. Moreover, the field of lung mechanobiology, which studies the role of physical forces and tissue properties in lung biology ${ }^{6}$, has long been puzzled by the apparent paradox that mechanical stresses are reported to stimulate lung growth ${ }^{7}$ and regeneration after injury ${ }^{8}$, but can also cause long-lasting damage in the context of mechanical ventilation ${ }^{9}$. It has been suggested that tissue stretch induces fibroblast and alveolar epithelial cell proliferation, whereas the effects of mechanical forces on cellular differentiation depend on many factors, including the cell type in question, gestational stages, previous injuries, and the experimental conditions ${ }^{10}$. For example, the tissue distention in the prenatal lung due to fluid pressure from fetal breathing movements and potentially smooth muscle contractions are critical to lung growth ${ }^{11,12}$. However, while artificially prolonging these strains promotes additional lung growth, alveolar differentiation is stunted ${ }^{13}$.

While these and other studies ${ }^{14-16}$ demonstrate the complex, and still mostly unresolved, effects of breathing-related forces on alveolar epithelial biology, even less is known about their contribution to airway epithelial biology, mainly due to the lack of experimental platforms allowing studies on welldifferentiated airway epithelial cell cultures at the air-liquid interface (ALI). Previous work has focused on the detrimental effects of mechanical ventilation ${ }^{17}$ or bronchoconstriction ${ }^{18}$, and employed whole airway tissue mounts ${ }^{19}$, undifferentiated bronchial epithelial basal cells ${ }^{20}$, or cell lines ${ }^{21}$. This leaves unresolved the fundamental question of whether healthy respiratory forces positively impact human airway epithelial biology. Here, we hypothesized that normal breathing-associated airflow shear and strain can modulate differentiation and homeostasis of primary human airway epithelial cells. We reasoned that since the human airway epithelium turns over every 30 to 50 days $^{4}$, airway epithelial cell differentiation might be responsive to the mechanical forces of postnatal breathing, i.e., at ALI. 
First, we focused on developing a human well-differentiated primary airway epithelial culture on an Organs-on-Chips (OOC) platform that provides microfluidic perfusion, including airflow, and the ability to cyclically stretch the cell culture membrane. Most current Airway Lung-Chip models use rigid polymer membranes as cell substrates, which excludes the use of breathing stretch ${ }^{22,23}$. Next generation $\mathrm{OOC}$ platforms, including the commercially available Emulate Chip-S1, overcome this issue by integrating a flexible membrane that supports dynamic stretch ${ }^{24,25}$. The $7-\mu \mathrm{m}$ pore size of the Chip-S1 membrane furthermore supports studying immune cell recruitment ${ }^{26}$. However, spontaneous transmembrane migration of airway epithelial cells might also occur ${ }^{27}$. A possible strategy is the concurrent seeding of epithelial and endothelial cells onto either side of the membrane to prevent such behavior ${ }^{28}$. Unfortunately, this method precludes the study of either compartment in isolation, constrains the type of media that can be used during epithelial differentiation, and prevents full differentiation of the epithelial cells before endothelial cells undergo senescence or suffer from suboptimal co-culture medium.

Here, we sought to optimize primary airway epithelial cell culture with optional co-culture of endothelial cells in the Chip-S1. We present a new Airway Lung-Chip protocol that uses a PDMS membrane functionalization strategy to enable robust epithelial and endothelial cell adhesion in the desired chip compartment while preventing migration into other compartments. We validated differentiation of the airway epithelial cell culture by comparison to established in vitro models in cell culture inserts. Leveraging this Airway Lung-Chip model we then tested our hypothesis and explored the effects of breathing-like biomechanical cues on airway epithelial cell biology. We evaluated genetic, cellular, and functional markers of differentiation and homeostasis of primary airway epithelial cells at ALI in response to laminar airflow and cyclic membrane strain at physiological levels.

\section{Results}

\section{Culturing primary Human Bronchial Epithelial Cells on a large pore-size PDMS membrane}

We utilized the Chip-S1 platform (Emulate Inc.) to assess how breathing-associated stimuli such as airflow and stretch affect airway epithelial biology. The two adjacent microchannels in the chip allow for dynamic flow of air in the apical, epithelial channel and continuous perfusion with cell culture medium in the basal channel that can also be used for endothelial co-culture. The channels are separated by a flexible, optically transparent PDMS membrane with well-defined 7- $\mu \mathrm{m}$ diameter pores that is suited for real-time imaging and that can be stretched in a controlled manner using vacuum actuation (Fig. S1A). This is in contrast to the rigid and translucent polyethylene terephthalate (PET) membranes with highly variable pore size used 
in previous Airway Lung-Chip studies and in classical cell culture inserts, also called Transwells (TW) $)^{22,23,29}$ (Fig. S1B). We encountered several challenges for using this chip design that seem to be specific to airway epithelial cell culture and not applicable to other organ systems on the Chip-S1 ${ }^{25,30-33}$. Primary human bronchial epithelial cells ( $\mathrm{PHBEC}$ ) failed to robustly adhere to the membrane when we tested the protocol previously developed for the Airway Lung-Chip with PET-membrane ${ }^{23}$. After testing a variety of commonly used coating compounds, we found that coating the activated membrane with either $300 \mu \mathrm{g} / \mathrm{mL}$ collagen IV or a $0.1 \%(\mathrm{w} / \mathrm{v})$ solution of bovine serum albumin (BSA) supported normal cell attachment and morphology. However, over the course of cell culture, cells migrated across the membrane to the basal channel and continued to proliferate until confluent and fully covering the membrane and walls, which we verified using our newly developed method of chip sectioning and imaging (Fig. 1A, left and Fig. S1C). The extent of migration was donor dependent and usually started within days after seeding up to the first week of differentiation at ALI. Basal channel invasion occurred in most chips and progressed significantly over time (Fig. 1A, center). The proliferating epithelial cell layer in the basal channel likely limited the availability of nutrients to the apical channel, such that the apical cell layer often degenerated and detached in the second week of ALI. When invasion was less severe, the pHBEC differentiated into multiciliated epithelia with functional mucus clearance (Fig. S1D-F), suggesting general suitability of the protocol and membrane to support airway epithelial culture.

To prevent migration of pHBEC to the basal channel during differentiation, a variety of strategies were evaluated, including the addition of signaling inhibitors to the medium and altering media composition (summarized in Table S1). None of these approaches significantly reduced invasion into the basal channel while preserving differentiation capacity (data not shown). We next created a mechanical barrier to invasion by covering the membrane in the apical channel with a variety of collagen-based threedimensional hydrogels before seeding the epithelial cells (Fig. S2A and B), using a similar gel deposition method we previously developed for sandwiching hepatocytes in a Liver-Chip model ${ }^{30}$. The epithelial cells attached to different gel types, and significant reduction in basal channel invasion was observed compared to chips without hydrogels (Fig. 1A, right). At day 14 of ALI a well-differentiated epithelium containing club, ciliated, goblet and basal cells had formed (Fig. S2C). While coverage by motile cilia and ciliary beat frequency ( $\mathrm{CBF}$ ) were lower in the hydrogel chips compared to BSA control chips for the single donor that was tested, the average speed of mucociliary clearance (MCC) showed no significant difference (Fig. S2D). However, given substantial variability between extracellular matrix (ECM) lots used for hydrogel preparation and other factors influencing spreading, gelling, and final geometry in the microfluidic channel, it was technically challenging to reliably deposit gels without any holes (that allowed migration). 
Therefore, while noting the potential future application of using the gel layer to embed stromal cell types, we pursued a different and more reproducible strategy to overcome the migration challenge.

The final solution to preventing migration of pHBEC into the basal channel was to functionalize the basal channel with the anti-fouling agent Pluronic F-127. Pluronic is a surfactant that reversibly binds to hydrophobic surfaces, such as PDMS, and prevents protein and cell adhesion ${ }^{34}$. Using this surface functionalization strategy, migration was significantly reduced (Fig. 1B). We confirmed the possibility to remove the Pluronic coating and establish co-culture with endothelial cells in the basal channel after pHBEC differentiation (see methods). Chips evaluated $48 \mathrm{~h}$ after seeding presented with confluent endothelial cells expressing key endothelial markers (Fig. S3). Together, these results demonstrated that the Pluronic membrane functionalization strategy allows for the robust and, importantly, reversible prevention of unwanted cellular adhesion into the basal channel. In the following, we returned to studying epithelial cell biology without endothelial co-culture.

\section{Comparison of pHBEC culture in Airway Lung-Chips and TWs}

Having resolved the migration issue, we next sought to assess the differentiation of pHBEC in the dynamically perfused chip model compared to established static TW culture models ${ }^{35}$. We found similar expression levels of cell-type specific genes in both conditions, except for cytokeratin 8, which characterizes the differentiated non-basal epithelial cells and was significantly lower in TW cultures compared to the chip (Fig. 1C). We confirmed these findings with confocal microscopy (Fig. 1D) and did not find any substantial differences between the luminal cell populations (i.e., ciliated, goblet and club cells) of Chip and TW cultures (Fig. 1E). 
A
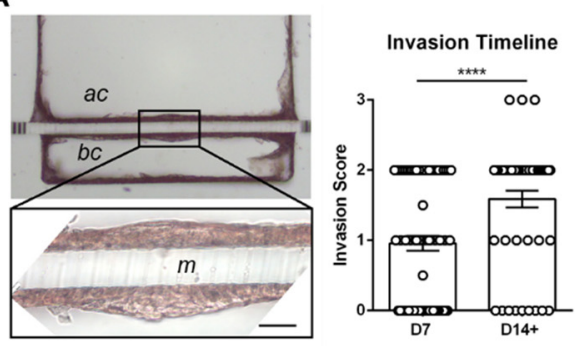

B

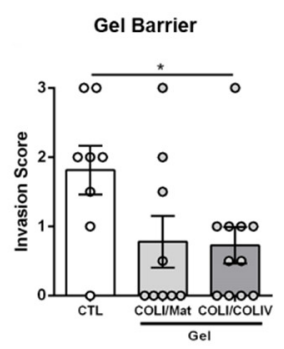

Pluronic Functionalization

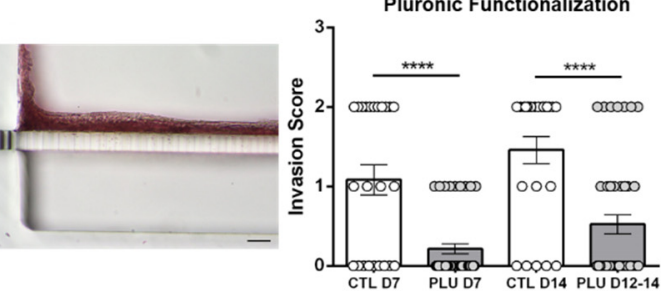

C
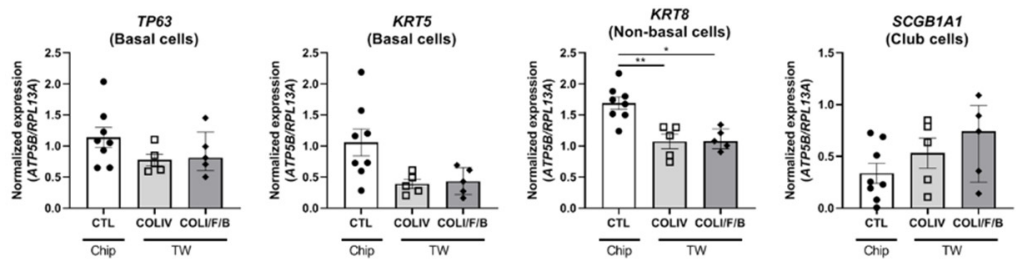

D
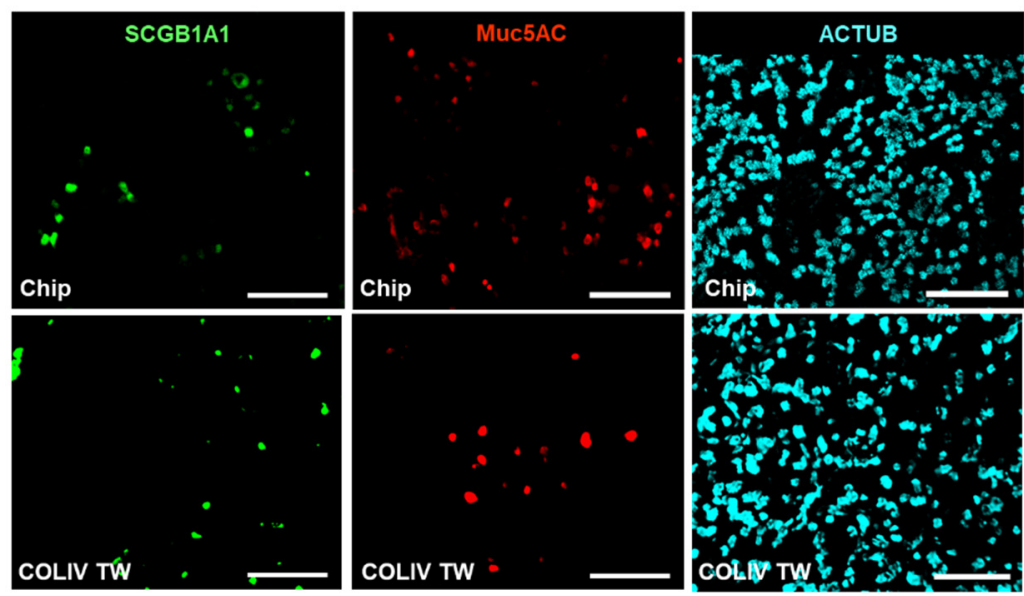

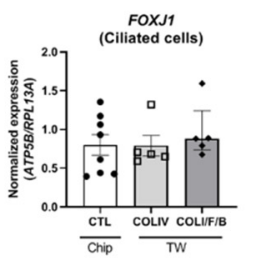

$\mathbf{E}$

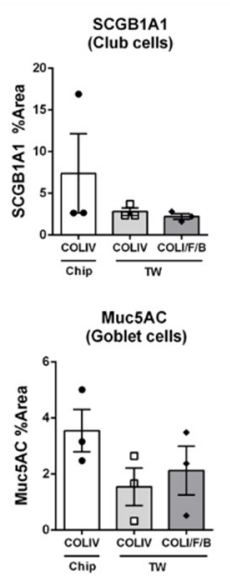

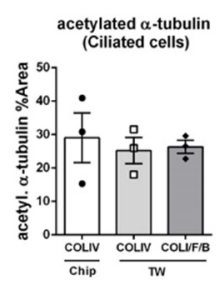

Figure 1: Optimization of airway epithelial cell differentiation on the Chip-S1.

A, Left: Histology cross-section of a fully invaded chip with pHBECs in both channels (H\&E stain). Ac, apical channel, bc, basal channel. Insert: Cells line both sides of the membrane $(\mathrm{m})$. Scalebar: $50 \mu \mathrm{m}$. Center: Quantification of basal channel invasion by pHBEC on day 7 and after day 14 of ALI using a score of 0 (no invasion) to 3 (basal channel fully lined). Data pooled from multiple media and membrane coating conditions. $\mathrm{N}=4$ donors with 2-6 chips each. Each data point is from 1 chip. Columns and error bars represent mean +/- SEM, $* * * *<0.0001$, Mann-Whitney test. Right: Reduced invasion at day 14 ALI in chips with collagen I/Matrigel (COLI/Mat) or collagen I/collagen IV (COLI/COLIV) hydrogels. N=1 donor; each data point is from 1 chip. Data are depicted as mean +/- SEM. ${ }^{*} p<0.05$, ANOVA, followed by Dunnett's multiple comparison test. B, Left: Representative image of chip cross-section with pHBECs in apical channel CH\&E staining). Scalebar: $50 \mu \mathrm{m}$. Right: Significantly reduced invasion in chips treated with Pluronic. Pluronic data are from $\mathrm{N}=3$ different donors, 12 chips each; CTL data are the same as in Figure $1 \mathrm{~A}$ center. Each data point is from 1 chip. Data are depicted as mean +/- SEM. ****, p<0.001, MannWhitney test. C, Gene expression of TP63 (basal cells), KRT5 (basal cells), KRT8 (differentiated non-basal cells), SCGB1A1 (club cells), MUC5AC (goblet cells) and FOXJ1 (ciliated cells) in collagen IV (COLIV)-coated chips and TWs or collagen I/Fibronectin/BSA (COLI/F/B)-coated TWs at day 14 ALI. Data are shown as target gene expression normalized for the geometric mean expression of the reference genes ATP5B and RPL13A; For the chips: $\mathrm{N}=8$ different donors, one chip each. For the TWs: $\mathrm{N}=5$ different donors, one TW each. Data are depicted as mean +/- SEM. *, $\mathrm{p}<0.05 ;{ }^{* *}, \mathrm{p}<0.01$, Mixed-effects analysis combined with a 
Dunnett's multiple comparisons test. D, Representative IF staining of goblet, club, and ciliated cells in chip and COLIV TW. Scalebar: $100 \mu \mathrm{m}$. E, Quantification of the cell culture surface area fraction positive for IF markers of each cell type. $\mathrm{N}=3$ donors; each data point is mean value from 1 donor. No statistical difference was detected using ANOVA followed by Dunnett's multiple comparison test.

\section{Effects of airflow and cyclic strain on pHBEC differentiation}

After establishing a robust and reproducible cell culture protocol for the Airway Lung-Chip, we next investigated the combined effect of airflow in the apical channel and physiological cyclic membrane strain $(\mathrm{AF} / \mathrm{S})$ on pHBEC differentiation compared to control (CTL) chips. Airflow in AF/S chips was set to $1.2 \mu \mathrm{l} / \mathrm{s}$, generating a shear stress of ca. $0.1 \mathrm{mPa}$ on the cells, which is on the same order of magnitude as estimated airflow shear stresses during tidal breathing ${ }^{36}$. The membrane of AF/S chips was actuated linearly and perpendicular to the channel at a rate of $0.25 \mathrm{~Hz}$ to achieve maximally $5 \%$ cyclic strain in the center of the chip. This mimics the estimated physiological mechanical strain in most of the airway tree during tidal breathing at rest ${ }^{1}$. We chose to apply AF/S between day 7 and 14 of $A L I$, after which chips were evaluated for cellular markers of differentiation. This protocol allowed for barrier formation before the onset of mechanical stresses and provided sufficient time for key cellular markers to be expressed in vitro ${ }^{35}$. First, we assessed if the pHBEC in the Airway Lung-chip had a classical stratification of basal cells close to the membrane, and differentiation with luminal cells located more apically and featuring ciliation. Therefore, we imaged the cross-section of the epithelial cell layer and confirmed that basal cells were localized close to the basolateral membrane and ciliated cells were visible on the apical surface (Fig. 2A). Next, we assessed the cellular composition of the epithelium using qPCR analysis of cell-type specific marker genes. Compared to CTL chips, AF/S chips expressed significantly lower levels of basal (TP63 and KRT5) and differentiated non-basal (KRT8) cell markers as well as near-significantly greater expression of SCGB1A1, a marker of club cells ( $p=0.055$ ) (Fig. 2B). The difference in gene expression may capture an early stage in club cell development as the cell culture-wide density of immunofluorescent (IF) labeled club cell protein was similar between groups (Fig. 2C and D). We verified that lactate dehydrogenase (LDH) levels, ciliary beat frequencies (CBF), and the speed of mucociliary clearance (MCC) were similar between both chip groups, indicating comparable levels of cell viability and cilia function (Fig. 2E-H). 
A
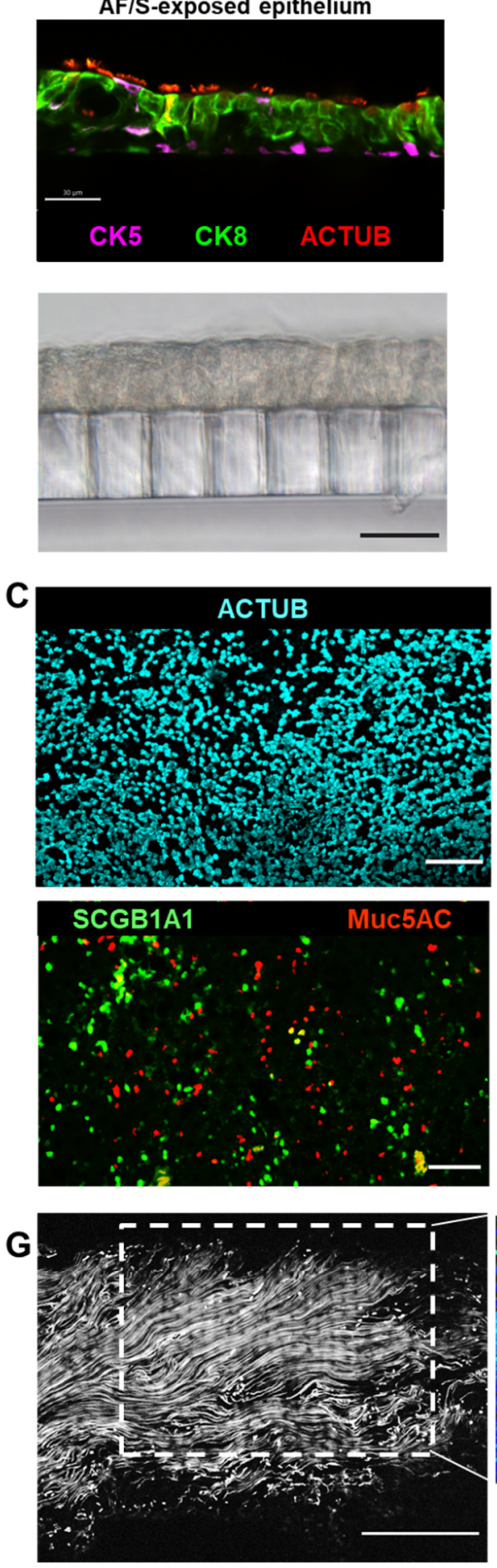

B
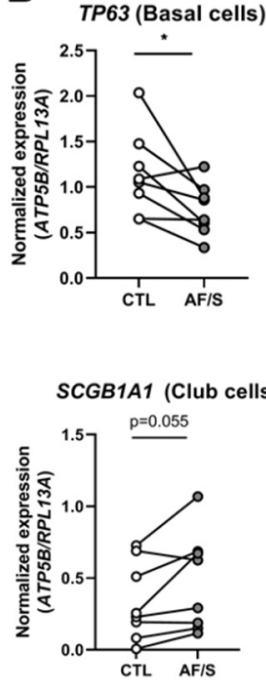

D

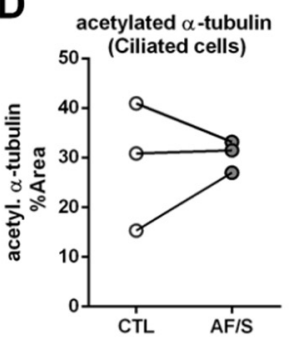

E
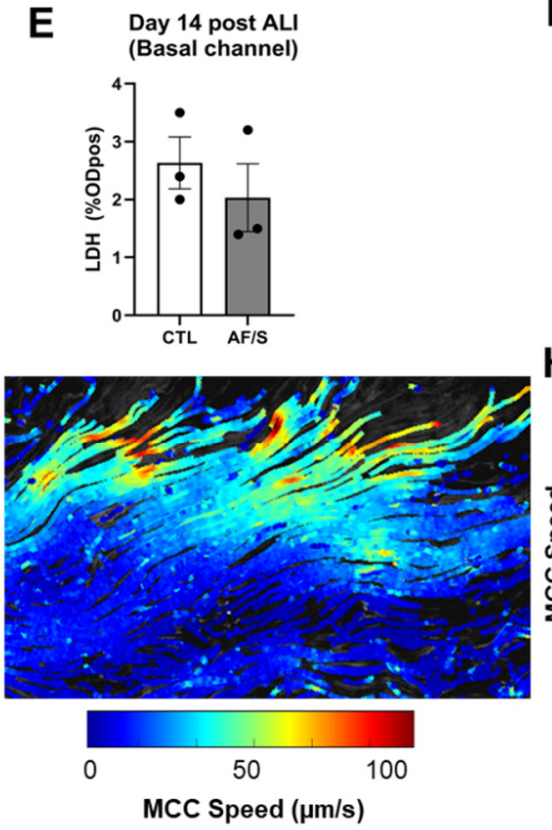
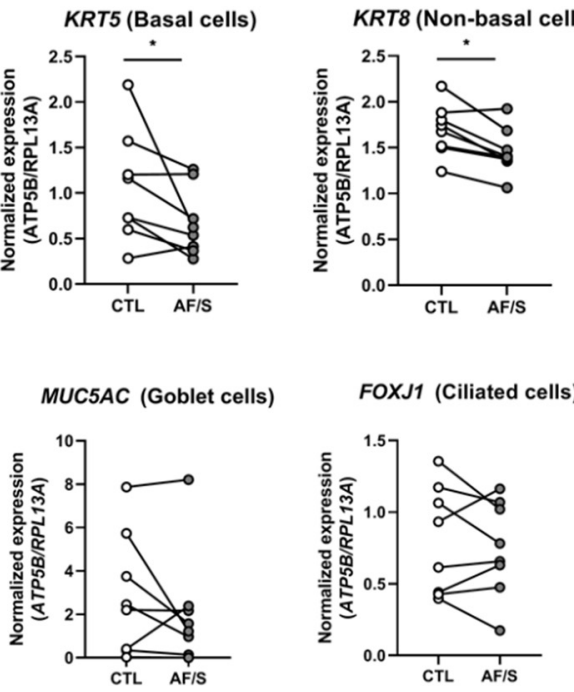

FOXJ1 (Ciliated cells)
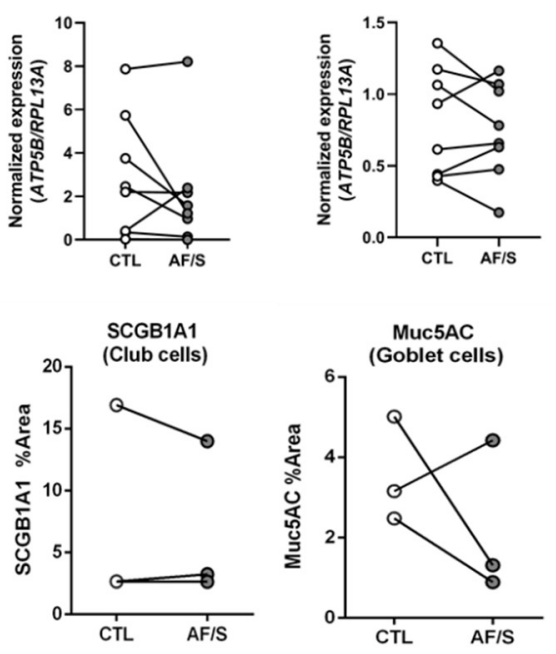

F

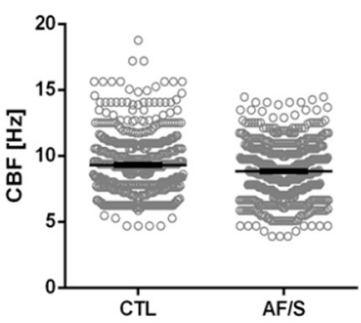

H

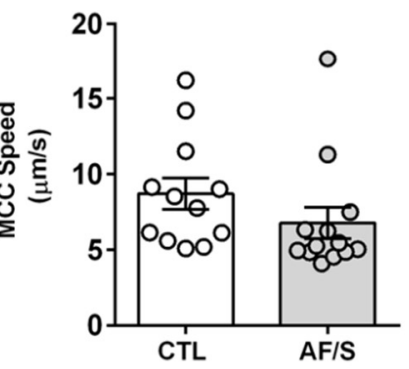


Figure 2. Comparison of cellular composition and cilia function between pHBEC cultured in the Airway Lung-Chip at day 14 of ALI with and without airflow and stretch treatment.

A, Immune fluorescent (IF) staining and brightfield image of AF/S chip cross section. Cytokeratin 5 (CK5; purple), cytokeratin 8 (CK8, green) and acetylated alpha-tubulin (ACTUB; red). B, Gene expression at day 14 ALI of TP63 (basal cells), KRT5 (basal cells), KRT8 (differentiated non-basal cells), SCGB1A1 (club cells), MUC5AC (goblet cells) and FOXJ1 (ciliated cells). Open circles: CTL chips, gray circles: AF/S-exposed chips; $\mathrm{N}=8$ different donors, one chip each. Data are depicted as mean with SEM. *, $\mathrm{p}<0.05$ as assessed by Wilcoxon matched-pairs signed rank test. C, Representative IF stains of epithelial cell types in AF/S chip. Top: cilia. Bottom: club and goblet cells. D, Quantification of the relative surface area labeled with each cell type marker. $\mathrm{N}=3$ donors; each point is mean value from 1 chip. $\mathrm{E}, \mathrm{LDH}$ analysis on medium collected from the basal channel reservoir after $24 \mathrm{~h}$ of flowing between day 13 and 14 of ALI. Data are from N=3 different donors, 1 chip each. $\mathrm{F}$, Comparison of CBF on chip. Data from N=1 Donor, 2-4 chips per condition. Each data point is approximately 1 ciliated cell. Column and error bars represent mean +/- SEM. G, Example of bead trajectories visualizing MCC on chip. Scalebar: $300 \mu \mathrm{m}$. Inset: Example of color-coded speed of MCC. H, Comparison of MCC speed. Data from N=3 Donors, 3-4 field of views (FOV) each. Each data point is mean value from 1 FOV. Column and error bars represent mean +/- SEM.

Transcriptomic analysis to probe effects of airflow and dynamic stretch on the airway epithelium

To further explore the effects of AF/S exposure on airway epithelial cell biology, we performed an unbiased bulk RNA-sequencing (RNAseq) analysis on RNA isolated from Airway Lung-Chip cultures from three different donors collected at day 14 of ALI, i.e., after 7 days of exposure to AF/S. We also included conventional PET membrane-based TW cultures with either the same COLIV coating as used in the chips or with a collagen I/fibronectin/BSA coating ${ }^{35,37}$ to compare this completely static system to the partly dynamic CTL chips (medium perfusion only) and the fully dynamic AF/S chips. Principal component analysis (PCA) revealed a pronounced difference between the transcriptional profiles of TW and chip cultures, and a smaller difference between donors (Fig. 3A). Applying AF/S to the chip cultures shifted the transcriptional profile further (Fig. 3A). We identified 21 significant differentially expressed (DE) genes between AF/S chips and CTL chips (Fig. 3B, Table 1). Pathways associated with these genes confirmed that airflow and stretch affected epithelial cell differentiation and development (Table 2). A larger difference was found between the gene expression profiles of chips and TWs. We identified 2264 DE genes between CTL chips and COLIV TWs, and 2869 DE genes between AF/S chip and COLIV TWs (Table S2). A subset of the DE genes found in the comparison of COLIV TWs and CTL chips was represented in multiple topassociated pathways, including TNF signaling via NFKB, intracellular signaling, and proliferation (Table S3). The expression pattern of this DE gene subset revealed clear differences in the epithelial biology of TWs compared to both CTL and AF/S chips (Fig. 3C). We were intrigued by the downregulation of PTGS2 by AF/S exposure as suggested by RNAseq (Fig. 3B) as well as qPCR analysis (Fig. 3D), since previous work had 
shown its upregulation in mechanically stressed lung epithelia ${ }^{38}$. Furthermore, whereas application of short-term, non-physiological strain levels increases the production of the inflammatory mediator IL-8 in lung epithelial cells ${ }^{39-41}$, we found that IL-8 protein secretion was similar in CTL and AF/S chips $24 \mathrm{~h}$ after start of AF/S application, and significantly lower in AF/S chips on day 5 and 7 of AF/S (Fig. 3E). Since we had confirmed that CTL and AF/S chips both exhibited normal morphologies, comparable ciliary function, and similarly low LDH levels (Fig. 2E), we concluded that the reduced IL-8 levels in AF/S chip cultures were not due to lower cell viability.

A

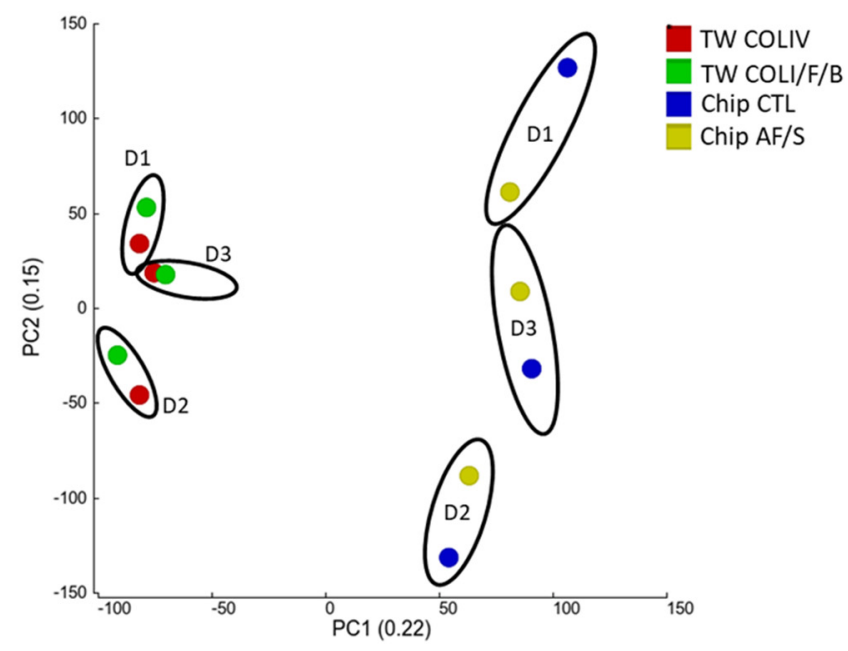

B

E

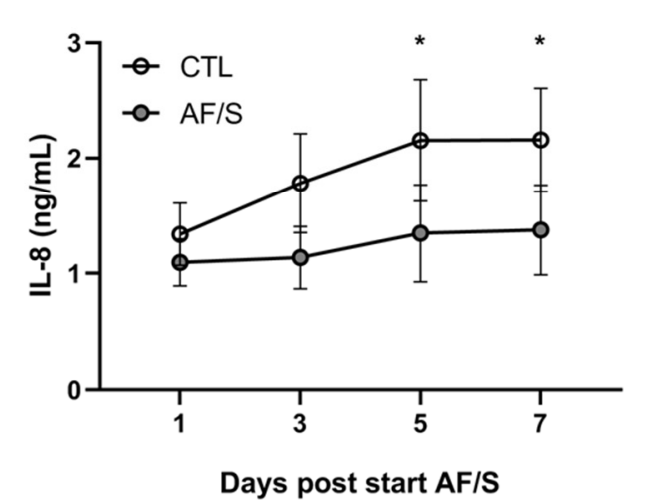

Days post start AF/S

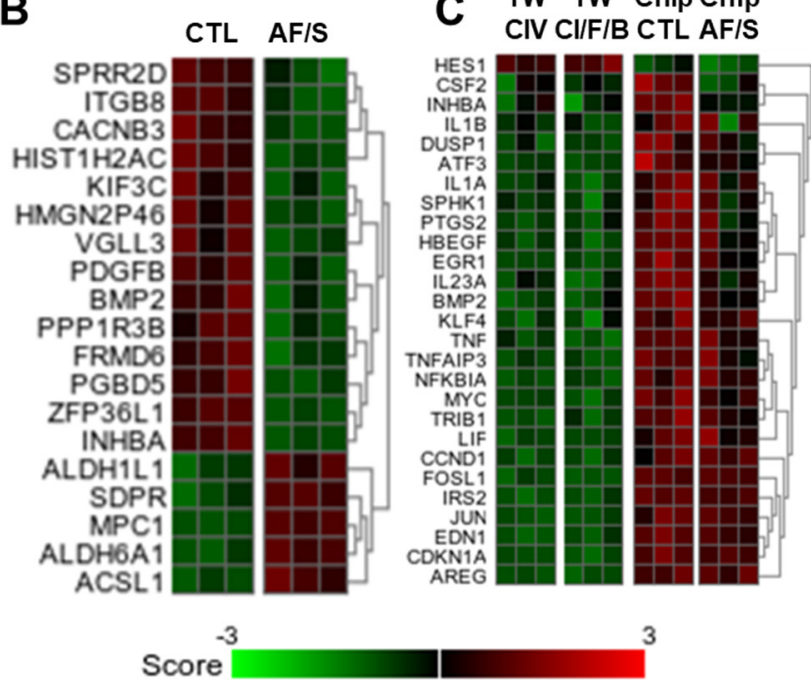

Day 7 post AF/S
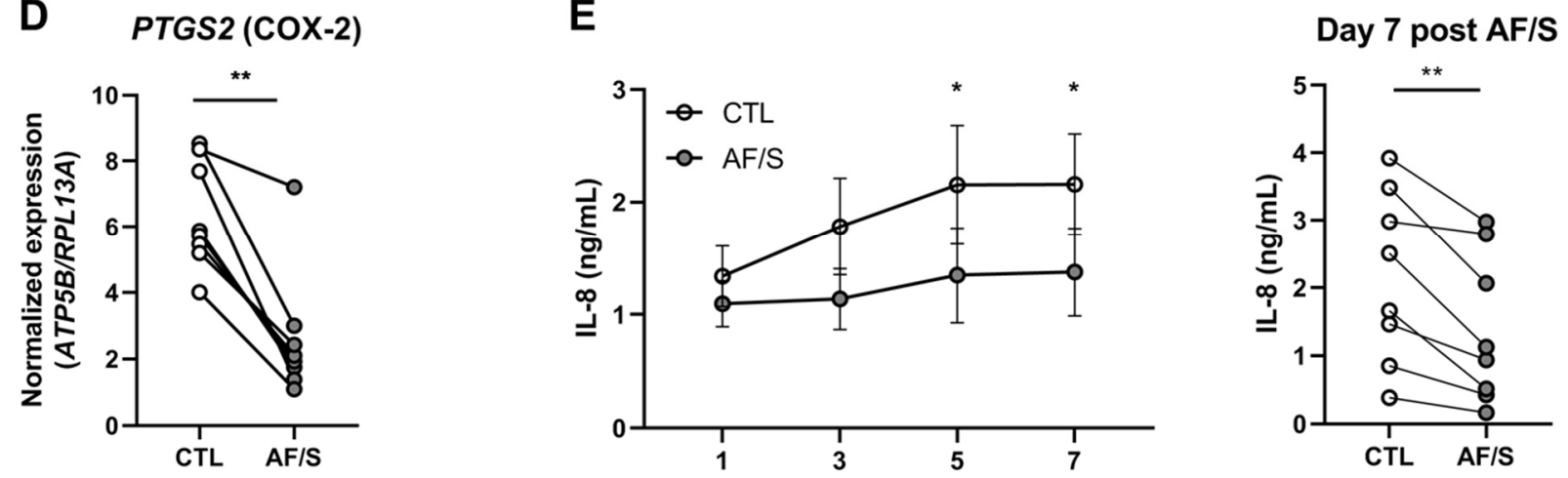

Figure 3: Comparison of RNAseq analysis of airflow and stretch-exposed Airway Lung-Chip cultures with control Airway Lung-Chip and TW cultures.

A, PCA on all transcriptomes showing distinct clusters of airway epithelial cells from donors $(N=3)$ in $C T L$, chips, AF/S chips, or TWs coated with collagen IV (COLIV) or with collagen I, Fibronectin, and BSA (COLI/Fib/BSA). B, Heat map displaying the Z score of all 21 DEGs between AF/S and CTL chip cultures. $\mathrm{N}=3$ different donors that are paired between $\mathrm{AF} / \mathrm{S}$ and $\mathrm{CTL}$ (one donor per column). C, Heat map displaying the $Z$ score of DEGs between CTL chip and COLIV (CIV) TW cultures that appeared most frequently in top pathways associated with the 2264 DEGs found in this comparison. D, Gene expression 
of PTGS2 at day 14 ALI. Open circles: CTL chips, gray circles: AF/S-exposed chips, $; ; \mathrm{N}=8$ different donors, one chip each. Data are depicted as mean +/- SEM. ${ }^{* *}, p<0.01$ as assessed by Wilcoxon matched-pairs signed rank test. E, Left: IL-8 protein levels (ELISA) in the basal channel medium collected $24 \mathrm{~h}$ after each medium change at the indicated times. Filled circles are CTL chips, open circles are AF/S chips; $N=8$ different donors, average of 2 chips each except for AF/S ( 2 donors; 1 chip each). Right: paired IL-8 levels at day 14 post $\mathrm{ALI}$ and 7 days of application of $\mathrm{AF} / \mathrm{S}$. $^{*}, \mathrm{p}<0.05$ as assessed by Mixed-effects analysis combined with a Sidak's multiple comparisons test. ${ }^{* *}, \mathrm{p}<0.01$ as assessed by a Wilcoxon test.

\section{Effects of Airflow and Stretch application on ECM-related gene expression by airway epithelial chip cultures}

Probing deeper into the potential effects of breathing-related forces on airway epithelial cell biology we explored if expression of ECM -related genes was changed in AF/S chips. Excessive mechanical stresses, such as those that occur during mechanical ventilation, have been shown to both alter the expression of ECM molecules and their breakdown, e.g. by matrix metalloproteinases (MMPs) ${ }^{42}$. Since the low donor number in our RNAseq analysis limited the statistical power and might mask relevant changes, we performed further analysis of multiple groups of ECM-related genes detected by the RNAseq analysis, i.e., collagens, glycoproteins (selection), SERPINS, MMPs, and LOXLs (Fig. 4A). We found that several major ECM(-related) genes, specifically MMP1, MMP9, COL1A1 (collagen type-1) and FN1 (Fibronectin), were downregulated by AF/S exposure in the RNAseq data across all donors, although not significantly $(\mathrm{N}=3$ ) (Fig. 4B). Subsequent $\mathrm{qPCR}$ analysis with $\mathrm{N}=8$ donors for MMP9 and FN1 confirmed the significant downregulation of both genes in AF/S-exposed cultures when compared to controls (Fig. 4C), validating the observations from the RNAseq analysis, and suggesting changes in ECM gene expression by AF/S exposure. 


\section{A}

Collagens

Glycoproteins

Serpins

MMPs
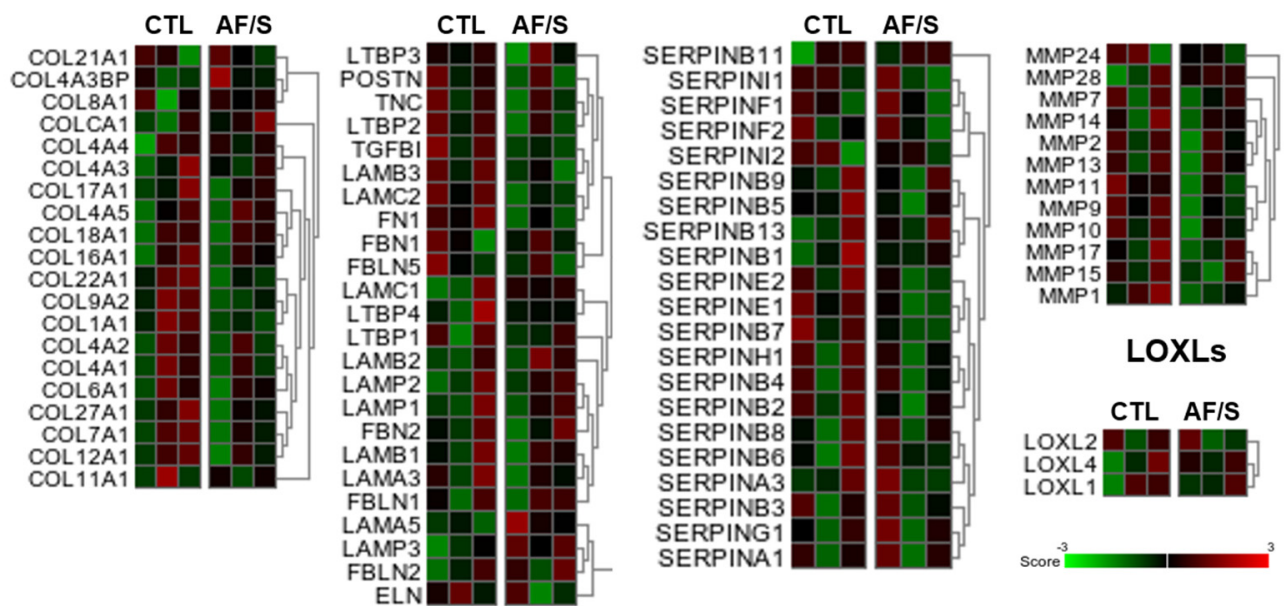

B COL1A1 (Collagen I)

FN1 (Fibronectin)

MMP1
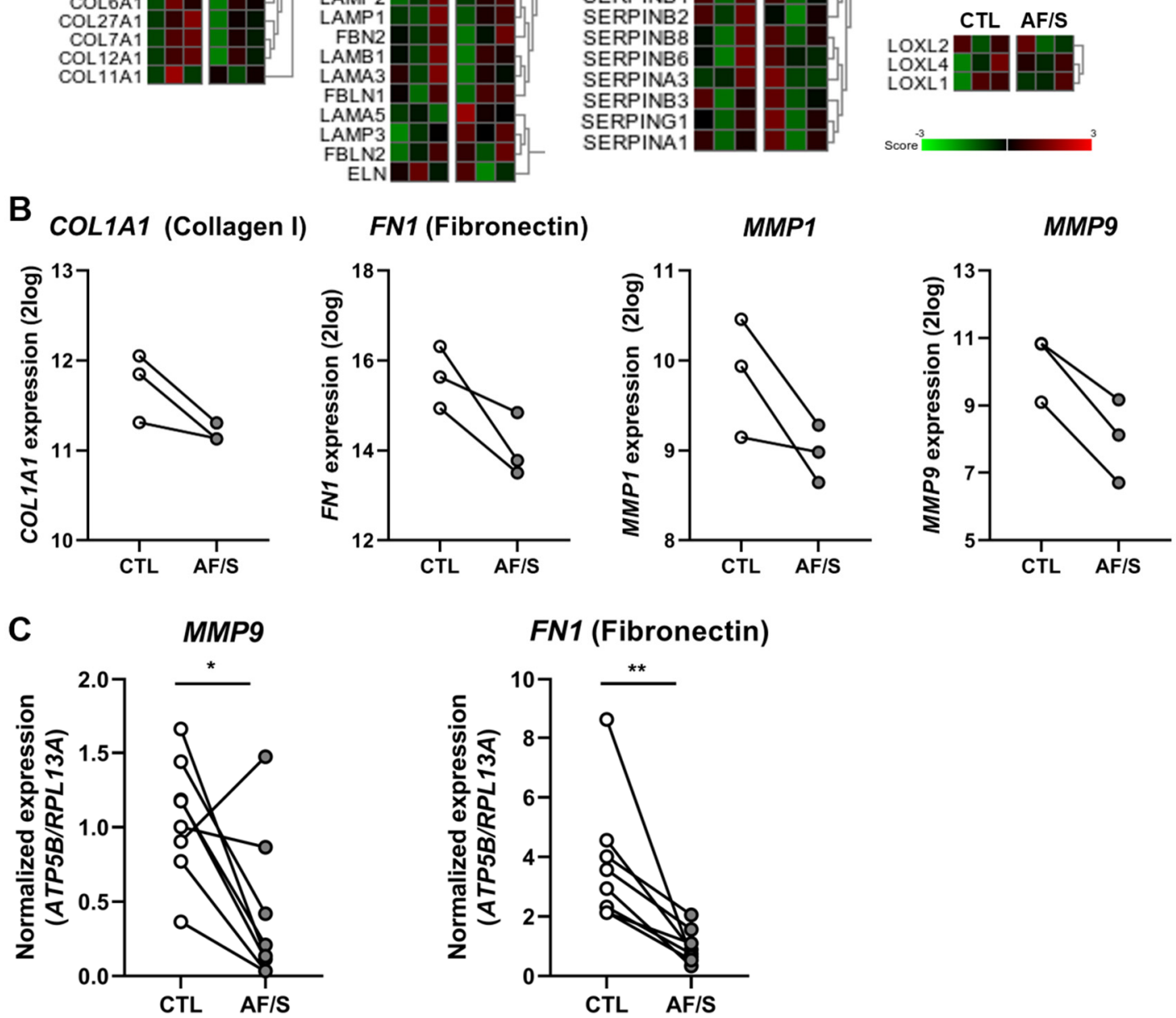

FN1 (Fibronectin)

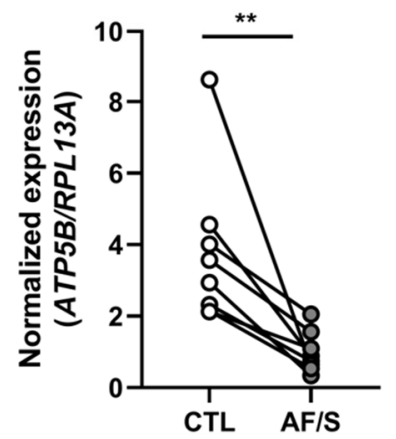

Figure 4. Effects of application of airflow and stretch to Airway Lung-Chip cultures on ECM-related genes.

A, Heat map of Z-scores for ECM-related gene expression (RNAseq), i.e., collagens, glycoproteins, matrix metalloproteinases (MMPs), SERPINs and LOXLs, in AF/S and CTL chips at day 14 of ALI. RNA was derived from 3 independent donors that are paired between AF/S and CTL (one column per donor)), 1 chip each. B, Donor-resolved gene expression (RNAseq) of selected genes shown in (A), i.e., collagen type-1 (COL1A1), fibronectin (FN), matrix metalloprotease 1 and 9 (MMP1 and MMP9) by AF/S and CTL chip cultures at day 14 of ALI. C, Gene expression assessed by qPCR analysis of MMP9 and FN1 at day 14 of ALI, 
Open circles: CTL chips, gray circles: AF/S chips.; ${ }^{*} p<0.05 ;{ }^{* *}, p<0.01$ as assessed by a Wilcoxon matchedpairs signed rank test, $\mathrm{N}=8$ different donors, one chip each.

\section{Discussion}

In this study we optimized culture of primary human airway epithelial cells in an Airway Lung-Chip model with a stretchable membrane. We subsequently used this model to study the influence of normal breathing-associated mechanical forces $(0.1 \mathrm{mPa}$ air flow shear stress and $5 \%$ cyclic strain) on airway epithelial cell biology and differentiation. Our key results showed that these cues alter the cellular composition and transcriptional profile of differentiating airway epithelial cell cultures. Specifically, we observed a reduction in the proportion of basal and differentiated non-basal cells and a near-significant increase in club cell marker in response to airflow and stretch, suggesting either increased levels of differentiation and/or transition to an epithelial cell-type composition that more closely resembles the smaller airways. Exposure to airflow and stretch furthermore elicited a reduction in both IL-8 secretion and in expression of genes related to inflammation and matrix remodeling. These effects of moderate levels of mechanical cues are in contrast with the upregulation of inflammatory markers reported previously in the context of mechanical ventilation and excessive mechanical stretch and compression ${ }^{9,18}$. Contrary to studies using whole mount tissue ${ }^{19}$, undifferentiated primary cells ${ }^{20}$ or cell lines ${ }^{21,39,43}$, our model may have captured phenotype-modulating, potentially even beneficial, effects of normal airflow and stretch by (i) using human primary cells from multiple human donors, (ii) allowing the basal cells to differentiate into a pseudostratified airway epithelium in the presence of mechanical stimulation, and (iii) applying mechanical stimulation over prolonged times, i.e. over 7 days compared to $24-48 \mathrm{~h}^{43,44}$, in order to capture adaptive remodeling rather than an acute insult. The temporal dynamics, remodeling processes, and heterogeneous responses as observed in our study would likely have been missed in these previously used experimental designs. Moreover, in the present study, we combined stretch application with airflow as both mechanical cues are intrinsically connected during breathing. Previous studies using airflow in airway cultures did not investigate effects on differentiation but focused for example on damage through cigarette smoke ${ }^{22}$, the deposition of particles ${ }^{45}$, and actin organization ${ }^{44}$. Taken together, our work addresses an important gap in our knowledge by investigating the long-time effects of normal breathing-associated forces on human airway epithelial differentiation, which thus far have remained understudied.

We selected the Chip-S1 platform for this study because it can deliver both linear airflow and cyclic strain while supporting endothelial co-culture and (immune) cell migration ${ }^{26}$. However, the comparatively large membrane pores also led to undesirable transmembrane cell migration during epithelial cell 
differentiation at ALI. We resolved this challenge using a surface functionalization strategy that controls cellular adhesion, enabling us to culture epithelial cells in the apical channel with or without co-culture of endothelial cells in the basal channel while maintaining the highly porous interface between the two compartments. Although we found that cell migration was not completely prevented in some donors, our data suggest a potential solution by increasing concentration or frequency of the Pluronic treatment.

To validate this Airway Lung-Chip model as an alternative to classical cell culture platforms, we assessed the airway epithelial cell composition in chips compared to TWs. Chip cultures tended to contain higher levels of basal and differentiated non-basal cells and lower levels of club cells, although the differences were not significant for all cell types. These results could indicate, although speculatively, that the epithelial composition on chips is more similar to those in the larger airways, whereas the epithelial composition in TWs may be closer to those found in small(er) airways ${ }^{4}$. Supporting this interpretation, larger airway epithelium generally has a thickness of $\sim 40-50 \mu \mathrm{m}^{46,47}$, similar to what we found in chip cultures and approximately twice the thickness of TW cultures in comparable culture medium ${ }^{48}$. Interestingly, when applying airflow and stretch to chip cultures we observed changes in epithelial cell composition that approached those in TWs. Together, these data may indicate that airflow and/or mechanical cues contribute to skewing epithelial cell composition towards a more small(er)-airway like phenotype characterized by reduced levels of basal cells, greater levels of club cells, and lower levels of goblet cells ${ }^{49}$. Though several studies have addressed cellular composition throughout the lungs ${ }^{50-52}$, data on regional cell-type composition and related gene expression signatures are limited. Frequently, a distinction is made only between large and small airways, overlooking the likely gradual change in epithelial phenotype from the large airways to the bronchioles. It will be important to quantify and characterize these different regions in human tissue in detail to have a reliable comparison of our cell cultures not only on chip but also in TW and organoid cultures. Such benchmark data would reveal whether in vitro models reflect any lung region-specific epithelial phenotypes and how this is affected by disease factors and culture conditions, including mechanical forces.

These effects of AF/S on epithelial differentiation could (also) be the result of an accelerated differentiation rate. For example, BMP2, a significantly differentially expressed gene between control and AF/S chips, was recently shown to have a dynamic expression pattern depending on differentiation status, with a relatively low expression in a healthy well-differentiated epithelium and higher expression levels during differentiation ${ }^{53}$. Expression of BMP2 was reduced in AF/S chips, potentially indicating that AF/S exposure may promote faster differentiation. It would be interesting to assess if BMP2 is downregulated in control chips at later time points to confirm this hypothesis. 
Our explorative RNAseq analysis generated interesting results but also lacked power as it only contained three donors, hence many genes may have changed without yielding significant differences compared to the control. We therefore explored ECM-related gene expression based on existing literature on the effects of mechanical stretch on epithelial cells. Interestingly, a study investigating the effect of cyclic stretch on 3D cultures of fetal rat lung cells found reduced fibronectin gene expression, similar to our results, but increased fibronectin protein levels ${ }^{54}$. Measuring protein levels in our cultures will therefore be highly relevant for further analysis. We also cannot exclude that changes in the gene expression profiles may be a consequence of changes in cell composition. For example, control chips displayed higher expression of MMP1, BMP2 and collagen, typically associated with basal cells ${ }^{55}$, which constituted a greater cellular fraction in control chips compared to AF/S chips.

A limitation of our study is that we terminated the cultures after 14 days at ALI, which allowed us to capture some differences in the rate of differentiation between conditions but may have resulted in missed effects that would emerge at later timepoints. For example, in assessing the effect of AF/S, we found no change in club cell protein levels whereas transcriptional levels were increased. This might be due to the comparatively slow rate at which club cells may mature compared to other major epithelial cell types ${ }^{56}$. Future studies are needed to understand the effect of AF/S on later stages of differentiation. In addition to limiting total culture duration, we also restricted application of stretch to a single level and duration and combined it with airflow, all for the practical reasons of limiting sample size while using multiple donors. For future studies, it would be highly relevant to investigate the isolated effect of airflow and stretch, and to test a range of strain levels. Previous studies on mechanical ventilation ${ }^{17}$, combined with our findings, suggest that the effects of stretch can be beneficial or harmful, depending on its levels. If such a dose-dependent relationship can be confirmed, it would resemble the findings in the alveolar epithelium where cyclic strains associated with mechanical ventilation (up to $30 \%$ strain) generate a range of damaging effects including cell death and the production of reactive oxygen species ${ }^{57,58}$, whereas physiological strain promotes alveolar epithelial type II differentiation compared to no $\operatorname{strain}^{16}$.

As the small airways experience greater levels of breathing strain ${ }^{1}$, applying different stretch intensities may also have powerful implications for modelling airway region-typical phenotypes and improving the maturation of stem cell-derived airway progenitor cells, and for our understanding of how mechanics shape the phenotypic landscape of the tracheobronchial tree ${ }^{49}$. Leveraging the co-culture protocol described in this study it also becomes possible to study how mechanical stretch and airflow affect the signaling between the epithelial and vascular compartment ${ }^{59}$. Moreover, our ability to add an ECM hydrogel to the apical channel opens the door to probe the effects of increased matrix stiffness, which is 
known to modulate pathological cell behavior in fibrosis ${ }^{60,61}$, and other matrix properties on both the epithelial cell layer and embedded fibroblasts in the context of mechanical strain.

Taken together, the new Airway Lung-Chip protocol presented here provides a dynamic alternative to the classical static TW model. In a proof-of-concept study, we demonstrate its use for revealing the role of breathing-like airflow and strains in shaping airway epithelial differentiation and homeostasis. Additionally, the chip's support of endothelial cell co-culture and immune cell migration opens the door to a myriad of applications in the study of airway tissue biology and disease.

\section{Acknowledgements}

We would like to acknowledge Sowmya Balasubramanian, Marianne Koliana, Ben Calvert and Erik Quiroz for their help with cell culture and chip maintenance. We thank the Emulate team, including Geraldine Hamilton, Carolina Lucchesi, Antonio Varone and Lorna Ewart, for support with Emulate equipment and technical discussions. We thank Lennart Voortman, Annelies Boonzaier-van der Laan and Mees de Graaf for testing imaging of the chip and training on the Dragonfly microscope. The work at LUMC is supported by an EU Marie Curie Global Fellowship (\#748569), the Dutch Society for the Replacement of Animal Testing (Stichting Proefdiervrij) and The Lung Foundation Netherlands (grant \#4.1.19.021). ALR is supported by the Hastings Foundation and Cystic Fibrosis Foundation (grants \#Firth17XX0 and \#21XX0).

\section{Methods}

Primary Human Bronchial Epithelial Cell Sourcing and Expansion

Leiden: Primary human bronchial epithelial cells (pHBEC) were isolated from macroscopically tumour-free resected lung tissue, expanded and stored at the Leiden University Medical Center, Leiden, the Netherlands as previously described ${ }^{37}$. Use of such lung tissue that became available for research within the framework of patient care was in line with the "Human Tissue and Medical Research: Code of conduct for responsible use" (2011) (www.federa.org), that describes the no-objection system for coded anonymous further use of such tissue. pHBEC were thawed in a T75 flask coated with $30 \mu \mathrm{g} / \mathrm{mL}$ bovine type-1 collagen (PureCol ${ }^{\circledR}$, Advanced BioMatrix, San Diego, CA), $10 \mu \mathrm{g} / \mathrm{mL}$ fibronectin (Promocell, PromoKine, Bio-connect, Huissen, The Netherlands) and $10 \mu \mathrm{g} / \mathrm{mL}$ BSA (ThermoFisher Scientific, 
Waltham, MA, USA) in BEpiCM-b basal medium (ScienCell Research Laboratories, Sanbio, Uden, The Netherlands), supplemented with $100 \mathrm{U} / \mathrm{mL}$ penicillin (Lonza, Verviers, Belgium), $100 \mu \mathrm{g} / \mathrm{mL}$ streptomycin (Lonza, Basel, Switzerland) and bronchial epithelial cell growth supplement (ScienCell). When reaching $\sim 90 \%$ confluency, cells were trypsinized in $0.03 \%(w / v)$ trypsin (ThermoFisher Scientific), 0.01\% (w/v) EDTA (BDH, Poole, UK), 0.1\% glucose (BDH) in phosphate buffered saline (PBS) and prepared for seeding. USC: Fresh pHBEC were isolated from lung explant tissue from rejected donor transplant from subjects with no prior evidence of chronic lung disease with IRB approval from the University of Southern California (IRB\# USC HS-18-00162) using established protocols ${ }^{62}$. Next, pHBEC were thawed in T75 flasks or $100 \mathrm{~mm}$ cell culture dishes coated with PureCol (Advanced Biomatrix, Carlsbad, CA, USA) using complete BEpiCMb basal medium as described above. When reaching $~ 90 \%$ confluency, cells were dissociated with Accutase (Sigma Aldrich, St. Louis, MO, USA) and prepared for seeding.

Emulate Inc.: Preliminary experiments were performed with pHBECs from a commercial supplier (Lifeline Cell Technologies, Frederick, MD, USA)

Airway Lung-Chip functionalization and cell culture

Airway Lung-Chip membrane activation: To hydrophilize the PDMS membrane, apical and basal channels of the Chip-S1 (Emulate, Inc., Boston, MA, USA) were filled with ER-1 solution (1 mg/mL in ER-2; Emulate Inc) followed by 10 minutes of UV-light exposure using a 36-Watt UV-chamber (NailStar Professional, Model NS-01-US). Next, channels were washed twice with ER-2 (Emulate Inc.). This ER-1/ER-2 procedure was repeated once more. After the second ER-2 wash, channels were washed and filled with cold PBS.

Airway Lung-Chip membrane coating: The chip apical channel was emptied and subsequently filled with $300 \mu \mathrm{g} / \mathrm{mL}$ human collagen IV (Sigma) solution in PBS. For optimization experiments, also other coatings were used: $0.1 \%$ BSA (Gibco, Waltham, MA, USA) or $30 \mu \mathrm{g} / \mathrm{mL}$ bovine type-1 collagen (PureCol ${ }^{\circledR}$, Advanced BioMatrix). Chips were placed in petri dishes containing a small, open container of PBS to promote humidity and kept overnight in the incubator at $37^{\circ} \mathrm{C} / 5 \% \mathrm{CO}_{2}$.

Epithelial cell seeding and submerged culture in the Airway Lung-Chip: The coating solution in the chip apical channel was removed and replaced with so-called B/D complete medium: a 1:1 mixture of BEpiCM$\mathrm{b}$ and DMEM medium (STEMCELL Technologies, Köln, Germany), supplemented with Bronchial Epithelial Cell Growth Supplement (ScienCell), and additional 50 nM EC-23 (Tocris, Bio-techne Ltd, Abington, UK); 25 mM HEPES (Cayman Chemical, Hamburg, Germany), $100 \mathrm{U} / \mathrm{mL}$ penicillin and $100 \mu \mathrm{g} / \mathrm{mL}$ streptomycin (ScienCell). B/D complete was pre-filtered using a $70 \mu \mathrm{m}$ cell strainer (BD Biosciences, Vianen, the 
Netherlands) or a $0.22 \mu \mathrm{m}$ vacuum filter unit (Steriflip ${ }^{\mathrm{TM}}$; Millipore, Sigma, Burlington, MA, USA) to remove any suspended particles that could block flow in the Chip-S1. Epithelial cells were seeded in B/D complete in the apical channel at $3 \times 10^{6}$ cells $/ \mathrm{mL}$ (ca. $90 \mathrm{~K}$ cells per Chip-S1, i.e., ca. $320 \mathrm{~K} / \mathrm{cm}^{2}$ ) and left to adhere in the incubator for 6h. At this point, the Pluronic treatment described below was applied to the basal channel in the final chip protocol. Next, chip channels were washed gently with pre-warmed B/D complete. The Airway Lung-Chips were connected to the pre-warmed media-filled fluidic manifolds, aka "Pods" (Emulate Inc.). After obtaining fluid connection between chips and Pods, these units were placed in the micro perfusion instrument, aka "Zoë" (Emulate Inc.). After finishing the initial regulate cycle program (which pressurizes the medium to increase gas solubility and remove nucleating air bubbles while the system calibrates), the Airway Lung-Chips were continuously perfused with a flow rate of $30 \mu \mathrm{l} / \mathrm{h}$ in both apical and basal channel. Approximately 24h after start of the first regulate cycle, a so-called via wash was performed, dislodging any bubbles in the Pod's reservoirs fluid vials, followed by a second regulate cycle. This was important to minimize the blockage of flow by air bubbles. When following this protocol, no degassing of medium was needed. Approximately 4 days after seeding (donor dependent), air-liquid interface (ALI) was established as described in the next paragraph.

Epithelial differentiation at air-liquid interface (ALI) in the Airway Lung-Chip: ALI was established by removing the medium from the apical channel and having only medium flowing through the basal channel. To equalize (hydrostatic) pressure at the membrane level, reservoirs in the Pod connecting the inlet and outlet of the apical channel were sealed with $1 \mathrm{~mL}$ B/D complete medium per reservoir. Nonetheless, occasional flooding of the apical channel would still occur, requiring daily monitoring. Submerged apical channels were emptied manually using a $1000 \mu \mathrm{l}$ pipette. Epithelial cultures were also monitored daily for flow issues and cellular invasion and morphology. If flow issues arose, chips were disconnected, channels were rinsed with medium, potential air bubbles were dislodged, and chips were re-reconnected after establishing liquid-liquid interface between chip and Pod. If this happened repeatedly in the same sample, the Pod was replaced. Every 48h, medium outlet reservoirs were emptied, and inlet reservoirs were filled with fresh medium.

\section{Migration prevention}

Hydrogel mechanical barrier: Different compositions of ECM gels were tested, including bovine type-1 collagen (FibriCol ${ }^{\circledR}$, Advanced BioMatrix), Matrigel ${ }^{\circledR}$ (Corning, Corning, NY, USA), Fibronectin (Gibco), collagen IV (Sigma), and the collagen cross-linking agent, microbial transglutaminase (MTG) (Modernist Pantry LLC, Eliot, ME, USA). All ECM mixtures were prepared in PBS- solution and contained the following: 
collagen I = $0.5 \mathrm{mg} / \mathrm{mL}$ collagen I; collagen I + MTG $=0.5 \mathrm{mg} / \mathrm{mL}$ collagen I $+4 \mathrm{mg} / \mathrm{mL} \mathrm{MTG}$; collagen I + Fibronectin $=0.5 \mathrm{mg} / \mathrm{mL}$ collagen I $+200 \mu \mathrm{g} / \mathrm{mL}$ Fibronectin; collagen I + collagen IV $=0.5 \mathrm{mg} / \mathrm{mL}$ collagen

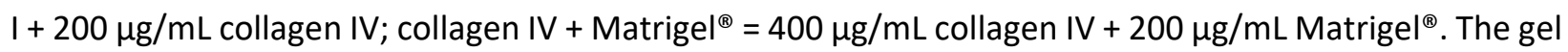
was prepared by injecting the gel prepolymer solution after membrane surface activation and incubated overnight at $37^{\circ} \mathrm{C}$. The next day, the gel was then flushed twice with $100 \mu \mathrm{L}$ of warm medium at 187.5 $\mu \mathrm{L} / \mathrm{s}$ flow (Eppendorf Xplorer automatic pipet; Eppendorf, Hamburg, Germany). This generated a 3D-ECM layer on the membrane with a thickness of 20-85 $\mu \mathrm{m}$. To assess and confirm ECM scaffold formation of different ECM compositions, we stained the gels with a fluorescent dye. Briefly, $1 \mathrm{mg} / \mathrm{mL}$ of $\mathrm{N}$ hydroxysuccinimide (NHS) ester dye (Atto 488 NHS Ester, Sigma Aldrich) was mixed with 50 mM borate buffer $(\mathrm{pH} 9)$ in 1:500 ratio. Directly after ECM formation, the prepared staining solution was injected into the apical channel and the chips were incubated for 25 minutes at room temperature in the dark. The apical channel was then rinsed three times with PBS prior to fluorescence imaging. Using Image ${ }^{63}$, the ECM-covered area was measured as a percentage to the total channel area via automated thresholding. The entire straight length of the channel was evaluated in all conditions ( $n=3$ chips each). To measure the ECM thickness, z-stack images with $4 \mu \mathrm{m}$ step size were acquired in two defined regions (left and right part) of the apical channel. Using ImageJ, the stacks were 3D-projected and rotated to obtain the $90^{\circ}$ - side view of the channel, followed by visualizing the stained ECM with different thicknesses via automated thresholding. Average ECM thickness was measured by calculating the threshold area divided by the length of the image.

Pluronic anti-adhesion surface treatment: Approximately $6 \mathrm{~h}$ after pHBEC seeding into the apical channel, the basal channels of all chips were coated with the surfactant Pluronic F-127 (Sigma) to prevent cell adhesion. For this, a minimum of $0.02 \% \mathrm{w} / \mathrm{v}$ Pluronic F-127 was freshly prepared in complete B/D medium and given time to dissolve at room temperature rather than at $37^{\circ} \mathrm{C}$, as solubility of Pluronic decreases with temperature ${ }^{64}$. Next, the solution was sterile filtered using a $0.22 \mu \mathrm{m}$ filter. The basal channel of the chips was emptied and filled with the Pluronic solution, followed by $1 \mathrm{~h}$ incubation at $37^{\circ} \mathrm{C}$. After the incubation, warm medium was used to gently rinse both channels before proceeding with connecting the chips to the perfusion instrument Zoë as described above. Our preliminary data suggest that the basal channel functionalization can be safely renewed at later timepoints by adding up to $0.2 \%$ Pluronic to the perfusion medium for 24 to $48 \mathrm{~h}$, which prevents basal channel invasion even in highly migratory donors. 
Primary Human Lung Microvascular Endothelial Cells (HLMVEC) were purchased from Cell Applications (San Diego, CA, USA) and expanded in Microvascular Endothelial Cell Medium (Cell Applications). Airway Lung-Chips were seeded with endothelial cells on day 11 or 12 of ALI. To prepare for endothelial cell seeding, the chips with epithelial tissue in the apical channel were disconnected from the Pods. Since Pluronic is more soluble in medium at lower temperatures ${ }^{64}$, we washed the basal chip channels at day 12 of ALI with ice-cold buffer. For this, the apical channel was filled with warm B/D complete medium, and the basal channel was incubated three times for 30 seconds, then rinsed, with ice-cold PBS to remove the Pluronic coating. The basal channel was filled with $100 \mu \mathrm{g} / \mathrm{mL}$ human fibronectin (Sigma) in B/D complete medium. The chips were flipped upside down, leveled and incubated for $1 \mathrm{~h}$ at $37^{\circ} \mathrm{C}$. Just before endothelial cell seeding, cells were washed with PBS and dissociated using Accutase (Sigma), then resuspended at $7.5 \times 10^{6}$ cells $/ \mathrm{mL}$ in endothelial cell medium. The fibronectin solution was removed from the basal channel and ca. $8 \mu$ l of the cell suspension was added, resulting in a seeding density of ca. 200, 000 cells $/ \mathrm{cm}^{2}$. The chip was again flipped upside down and left undisturbed in the incubator for $2 \mathrm{~h}$. After $2 \mathrm{~h}$, the endothelial cell medium was carefully exchanged in the basal channel. The chip was reconnected to the Pod and brought to ALI using standard procedures. The basal channel was perfused with a mix of $50 \%$ endothelial cell medium and 50\% B/D complete medium at standard flow rates $(30 \mu \mathrm{l} / \mathrm{h})$.

\section{Airway Lung-Transwell cultures}

Leiden: To match the seeding conditions on the chips, pHBEC were seeded at high density $(150,000$ cells/well) on ECM-coated $12 \mathrm{~mm}$ clear polyester Transwell inserts with $0.4 \mu \mathrm{m}$ pore size (Corning Costar, Cambridge, USA). TWs were either coated with $30 \mu \mathrm{g} / \mathrm{mL}$ Purecol (Advanced BioMatrix,), $10 \mu \mathrm{g} / \mathrm{mL}$ fibronectin (Promocell) and $10 \mu \mathrm{g} / \mathrm{mL}$ BSA (ThermoFisher Scientific) in PBS, or $300 \mu \mathrm{g} / \mathrm{mL}$ human collagen IV (Sigma) solution in PBS. Seeded cells were cultured submerged in B/D complete with EC-23 (50 nM). When cells were confluent, medium on the apical side was removed to initiate differentiation at air-liquid interface. Medium was changed 3 times a week and the apical side was washed with warm PBS.

USC: Clear polyester $6.5 \mathrm{~mm}$ Transwell inserts with $0.4 \mu \mathrm{m}$ pore size (Corning Costar, Cambridge, USA;) were pre-coated with $300 \mu \mathrm{g} / \mathrm{mL}$ human collagen IV (Sigma) solution in PBS.

HBEC were seeded at high density (70K cells/well) and cultured as above.

\section{RNA isolation and bulk RNA sequencing}

At day 14 of ALI, cells in the Airway Lung-Chips were lysed by disconnecting the chip from the Pod, lodging an empty filter tip at the outlet of the chip, and pipetting $100 \mu$ lysis buffer (Promega) with another filter 
tip into the inlet of the chip. This tip was then lodged into the inlet, such that the two tips inserted at either channel end were capturing the overflowing liquid. When visual inspection indicated lysis of the cells, the lysis solution was collected in a tube. Another $100 \mu \mathrm{l}$ was used to rinse the channel once more, to obtain in total $200 \mu \mathrm{l}$ of lysis solution from one chip. The lysis solution was stored at $-20^{\circ} \mathrm{C}$ until RNA extraction. Total RNA was robotically extracted using the Maxwell tissue RNA extraction kit (Promega) and quantified using the Nanodrop ND-1000 UV-Vis Spectrophotometer (Nanodrop technologies, Wilmington, DE, USA). Next, RNA was sent to GenomeScan (Leiden, the Netherlands) for Bulk RNA sequencing, or converted to CDNA for qPCR analysis. RNA sequencing was performed with the cDNA fragment libraries by the Illumina NovaSeq6000 sequencer using 150 bp pair-end sequencing settings.

\section{RNAseq analysis}

Data processing: Data processing was performed by GenomeScan. For this, the NEBNext Ultra II Directional RNA Library Prep Kit for Illumina was used to process the samples. The sample preparation was performed according to the protocol "NEBNext Ultra II Directional RNA Library Prep Kit for Illumina" (NEB \#E7760S/L). Briefly, mRNA was isolated from total RNA using the oligo-dT magnetic beads. After fragmentation of the mRNA, a cDNA synthesis was performed. This was used for ligation with the sequencing adapters and PCR amplification of the resulting product. The quality and yield after sample preparation were measured with the Fragment Analyzer. The size of the resulting products was consistent with the expected size distribution (a broad peak between 300-500 bp). Clustering and DNA sequencing using the NovaSeq6000 was performed according to manufacturer's protocols. A concentration of $1.1 \mathrm{nM}$ of DNA was used. Sequence reads were trimmed to remove possible adapter sequences using cutadapt v1.10. Presumed adapter sequences were removed from the read when the bases matched a sequence in the adapter sequence set (TruSeq adapters). For each sample, the trimmed reads were mapped to the human GRCh37.75 reference sequence (Homo_sapiens.GRCh37.75.dna.primary_assembly.fa). The reads were mapped to the reference sequence using a short-read aligner based on Burrows-Wheeler Transform (Tophat v2.0.14) with default settings. Based on the mapped locations in the alignment file the frequency of how often a read was mapped on a transcript was determined with HTSeq v0.11.0. The hit counts were summarized and reported using the gene_id feature in the annotation file. Only unique reads that fall within exon regions were counted. To enable comparison of gene/transcript expression across all samples outside of the context of differential expression analysis, RPKM/FPKM (reads/fragments per kilobase of exon per million reads mapped), TPM (transcripts per million), and CPM values were calculated with Cufflinks v2.2.1. 
Differential expressed genes: The read counts were used in the DESeq2 v2-1.14 package, a statistical package within the R platform R v3.3.0 to establish differentially expressed genes. Since groups had a small sample size and over-dispersion, an expression curve model based on a negative binomial distribution and local regression was used to estimate the relationship between the mean and variance of each gene. For heat maps, log2 expression of read counts are normalized with Z scores calculated by: (X (value of the individual sample) - $\mu$ (average of the row)) / $\sigma$ (standard deviation of the row).

Gene set analysis: The gene sets of differentially expressed genes were analysed in GSEA (Geneset Enrichment analysis) which also shows the related pathways. The significantly up- and downregulated genes (False Discovery Rate $(F D R)<0.05$ ) and related pathways between $C T L$ and $A F / S$ chips and between CTL chip and COLIV TW are provided in Table 1 and 2, and in Table S2 and S3, respectively.

\section{$c D N A$ and $q P C R$}

For cDNA conversion, 500 ng of total RNA was reverse transcribed using oligo dT primers (Qiagen Benelux B.V., Venlo, The Netherlands) and M-MLV Polymerase (Thermo Fisher Scientific) at $42^{\circ} \mathrm{C}$. All quantitative PCR (qPCR) reactions were performed in triplicate on a CFX-384 Real-Time PCR detection system (Bio-Rad Laboratories, Veenendaal, The Netherlands), using primers shown in Table S4 and IQ SYBRGreen supermix (Bio-Rad). The relative standard curve method was used to calculate arbitrary gene expression using CFXmanager software (Bio-Rad). Two reference genes were included to calculate the normalized gene expression.

\section{CXCL8 ELISA}

The protein encoded by CXCL8 (interleukin-8 or IL-8), was measured with use of the Recombinant Human IL-8/CXCL8 Protein ELISA kit of R\&D (Abingdon, UK) according to manufacturer's instructions and analyzed with use of 4-parameter logistic curve fitting in Prism 8 (version 8.1.1).

\section{Migration scoring}

We assigned the following scores to the degree of cell invasion into the basal channel, as assessed by phase contrast microscopy: 0 , no invasion; 1 , individual cells in the basal channel; 2 , confluent cell layers in the basal channel; 3, confluent tissue with multiple layers and/or fibroblast-like cell morphology in the basal channel.

IF staining and histology 
Leiden: At 14 day of ALI, cells in the Airway Lung-Chips were fixed using 4\% paraformaldehyde (PFA) solution. The PFA solution was pipetted into both channels and incubated for 20 min at RT, washed with PBS and stored in PBS at $4^{\circ} \mathrm{C}$ until staining. chips were subsequently removed from the carrier and cells were blocked and permeabilized using 0.5\% (v/v) Triton-X 100 in PBS with 5\% BSA for 60 min at RT. chips were cut into 2 pieces using a razor blade and channels were filled with primary antibodies (Table S5) diluted in the Triton/BSA buffer and incubated for $1 \mathrm{~h}$ at RT. Samples were rinsed three times $5^{\prime}$ with PBS. Next, channels were filled with secondary antibodies diluted in Triton/BSA buffer which was left incubating for $1 \mathrm{~h}$ at RT, followed by a triple $5 \mathrm{~min}$ wash with PBS. 4',6-Diamidino-2-phenylindole (DAPI, Sigma) was used to stain cell nuclei. Channels were next filled with prolong gold anti-fade (Thermo Scientific) and stored in the dark at $4{ }^{\circ} \mathrm{C}$ until imaging. Chips were imaged on bellco coverslips, $25 \times 75 \mathrm{~mm}$ (Electron Microscopy Sciences, Hatfield, PA, SUA) with 0.13-0.17 mm thickness using a Leica DMi8 microscope equipped with an Andor Dragonfly 200 spinning disk confocal using a 10x objective (NA 0.30), 20x water objective (NA 0.50 ) or $40 x$ water objective (NA 0.80 ).

USC: chips were fixed and stained as above and imaged on a Leica SP8 with HyVolution confocal microscopy imaging system using a 10x objective (NA 0.30).

\section{Cross-sectional imaging of thin chip slices}

A new sectioning technique was developed to obtain cross-sectional slices of the chip for histology and immunofluorescence staining. Chips were fixed as described as above. The PDMS surrounding the chip channel was cut away as much as possible using a razor blade. Next, the chip channel was mounted on the specimen tube using glue (Pattex instant glue) of a semi-automated vibrating microtome (Compresstome ${ }^{\circledR}$ VF-300-0Z, CMA, Kista, Sweden) and placed in the tube holder. Next, $150 \mu \mathrm{m}$ thick slices were cut and collected in PBS-filled wells of a 12-well plate. Slices were stained in the well for classical H\&E or immunofluorescence as described above. Slices were imaged on bellco coverslips as described above for the complete chip.

\section{IF Image processing and quantifications}

Raw images were split into individual channels, median filtered, cropped and CLAHE-filtered ${ }^{65}$ to remove illumination artefacts, and auto-thresholded to retrieve the area fraction positive for each cell marker. Semi-automated batch processing was conducted using ImageJ-Fiji ${ }^{66}$. 
Ciliary beat frequency. Ciliary beat frequency was measured using high-speed video microscopy followed by signal processing of the recordings as described previously in detail ${ }^{22}$. Briefly, to optimize optical imaging quality, inserts were washed for 10 minutes with PBS and the returned to ALI whereas Lung-Chips kept submerged in PBS or culture medium during imaging. Samples were imaged using a Leica DMi8 microscope equipped with phase-contrast objectives and a PCO Edge 4.2 high-speed camera with camera link interface that was operated from a PC desktop computer using the ImageJ-Fiji66 with MicroManager ${ }^{67}$. Per sample, 6 to 8 fields of view $(166 \times 166 \mu \mathrm{m}$, with ca. 3 pixel/ $\mu \mathrm{m}$ spatial resolution) were recorded under Koehler illumination at a temporal resolution of 200 frames per second. Using a custom algorithm in Matlab (MathWorks, Natick, MA, USA), regions containing ciliary beat activity were detected automatically by computing the standard deviation of each pixel's intensity over time, which reveals motion, and thresholding the resulting image using Otsu's method to segment area with detected motion. Ciliary beat density was computed by dividing the number of pixels with detected motion by the total number of pixels in the image. In regions with ciliary beat, the dominant beat frequency was identified from the smoothed pixel intensity over time using Fast Fourier Transform (FFT). The ciliary beat frequencies and ciliary beat densities measured in all samples per condition were pooled for statistical analysis.

Ciliary mucus clearance. Cilia-driven mucus transport was measured by seeding the samples with fluorescent tracer particles, recording the movement of the tracers over time using video microscopy, and tracking the displacement of the particles in the resulting videos using specialized software to derive transport velocities. Briefly, the samples were washed and prepared as above before adding $30 \mu \mathrm{l}$ of a 1:200 dilution of 1- $\mu \mathrm{m}$ sized fluorescent polystyrene microspheres (ThermoFisher Scientific) in PBS. Samples were imaged using a Leica DMi8 microscope equipped with a fluorescence light source and appropriate filters. Per sample, 6-8 fields of view (ca. 300×300 $\mu \mathrm{m}$ up to $1200 \times 1200 \mu \mathrm{m}$ ) were recorded for 10 to 20 seconds at a temporal resolution of 25 frames per second. Using a batch-processing script in ImageJ-Fiji with the Trackmate plugin ${ }^{68}$, we tracked the bead displacement per time step. Matlab was used to compute the mean transport speed for each bead and per field of view and for plotting the trajectories. Data from all samples per condition were pooled for statistical analysis.

\section{Statistical methods}

Statistical analysis was conducted using GraphPad Prism version 6 and 8 (GraphPad Software Inc., La Jolla, CA, U.S.A.). Differences were considered significant at $p<0.05$. Statistical significance, statistical methods, and the number of donors and chips used are indicated in the figures and figure legends. 


\section{References}

1. LaPrad, A. S., Lutchen, K. R. \& Suki, B. A Mechanical Design Principle for Tissue Structure and Function in the Airway Tree. PLOS Computational Biology 9, e1003083 (2013).

2. Roan, E. \& Waters, C. M. What do we know about mechanical strain in lung alveoli? Am J Physiol Lung Cell Mol Physiol 301, L625-L635 (2011).

3. Tsega, E. G. Computational Fluid Dynamics Modeling of Respiratory Airflow in Tracheobronchial Airways of Infant, Child, and Adult. Computational and Mathematical Methods in Medicine 2018, e9603451 (2018).

4. Crystal, R. G., Randell, S. H., Engelhardt, J. F., Voynow, J. \& Sunday, M. E. Airway Epithelial Cells. Proc Am Thorac Soc 5, 772-777 (2008).

5. Plasschaert, L. W. et al. A single-cell atlas of the airway epithelium reveals the CFTR-rich pulmonary ionocyte. Nature 560, 377-381 (2018).

6. Tschumperlin, D. J., Boudreault, F. \& Liu, F. Recent Advances and New Opportunities in Lung Mechanobiology. J Biomech 43, 99 (2010).

7. Nardo, L., Maritz, G., Harding, R. \& Hooper, S. B. Changes in lung structure and cellular division induced by tracheal obstruction in fetal sheep. Exp Lung Res 26, 105-119 (2000).

8. Liu, Z. et al. MAPK-Mediated YAP Activation Controls Mechanical-Tension-Induced Pulmonary Alveolar Regeneration. Cell Reports 16, 1810-1819 (2016).

9. Beitler, J. R., Malhotra, A. \& Thompson, B. T. Ventilator-Induced Lung Injury. Clin Chest Med 37, 633646 (2016).

10. Liu, M. \& Post, M. Invited Review: Mechanochemical signal transduction in the fetal lung. Journal of Applied Physiology 89, 2078-2084 (2000). 
11. Harding, R. Fetal pulmonary development: the role of respiratory movements. Equine Veterinary Journal 29, 32-39 (1997).

12. Schittny, J. C., Miserocchi, G. \& Sparrow, M. P. Spontaneous peristaltic airway contractions propel lung liquid through the bronchial tree of intact and fetal lung explants. Am. J. Respir. Cell Mol. Biol. 23, 11-18 (2000).

13. Joe, P. et al. Effects of mechanical factors on growth and maturation of the lung in fetal sheep. Am J Physiol 272, L95-105 (1997).

14. Edwards, Y. S., Sutherland, L. M., Power, J. H., Nicholas, T. E. \& Murray, A. W. Cyclic stretch induces both apoptosis and secretion in rat alveolar type II cells. FEBS Lett 448, 127-130 (1999).

15. Hammerschmidt, S. et al. Mechanical stretch alters alveolar type II cell mediator release toward a proinflammatory pattern. Am J Respir Cell Mol Biol 33, 203-210 (2005).

16. Sanchez-Esteban, J. et al. Mechanical stretch promotes alveolar epithelial type II cell differentiation. J. Appl. Physiol. 91, 589-595 (2001).

17. Nickles, H. T. et al. Mechanical ventilation causes airway distension with proinflammatory sequelae in mice. American Journal of Physiology-Lung Cellular and Molecular Physiology 307, L27L37 (2014).

18. Tschumperlin, D. J. \& Drazen, J. M. Feeling the squeeze: does airway constriction stimulate the bronchial epithelium? in Proceedings of the Second Joint 24th Annual Conference and the Annual Fall Meeting of the Biomedical Engineering Society] [Engineering in Medicine and Biology vol. 2 14681469 vol.2 (2002).

19. Le Guen, M. et al. The impact of low-frequency, low-force cyclic stretching of human bronchi on airway responsiveness. Respiratory Research 17, 151 (2016).

20. Waters, C. M. \& Savla, U. Keratinocyte growth factor accelerates wound closure in airway epithelium during cyclic mechanical strain. J Cell Physiol 181, 424-432 (1999). 
21. Doryab, A. et al. A Bioinspired in vitro Lung Model to Study Particokinetics of Nano-

/Microparticles Under Cyclic Stretch and Air-Liquid Interface Conditions. Front Bioeng Biotechno/ 9, 616830 (2021).

22. Benam, K. H. et al. Matched-Comparative Modeling of Normal and Diseased Human Airway Responses Using a Microengineered Breathing Lung Chip. Cell Systems 3, 456-466.e4 (2016).

23. Nawroth, J. C. et al. A Microengineered Airway Lung Chip Models Key Features of Viral-induced Exacerbation of Asthma. Am J Respir Cell Mol Biol 63, 591-600 (2020).

24. Stucki, J. D. et al. Medium throughput breathing human primary cell alveolus-on-chip model. Sci Rep 8, 14359 (2018).

25. Kasendra, M. et al. Development of a primary human Small Intestine-on-a-Chip using biopsyderived organoids. Scientific Reports 8, 2871 (2018).

26. Huh, D. et al. Reconstituting Organ-Level Lung Functions on a Chip. Science 328, 1662-1668 (2010).

27. Wesley, U. V., Bove, P. F., Hristova, M., McCarthy, S. \& van der Vliet, A. Airway epithelial cell migration and wound repair by ATP-mediated activation of dual oxidase 1. J Biol Chem 282, 32133220 (2007).

28. Si, L. et al. A human-airway-on-a-chip for the rapid identification of candidate antiviral therapeutics and prophylactics. Nature Biomedical Engineering 1-15 (2021) doi:10.1038/s41551-02100718-9.

29. Benam, K. H. et al. Small airway-on-a-chip enables analysis of human lung inflammation and drug responses in vitro. Nature Methods 13, 151-157 (2016).

30. Nawroth, J. C. et al. Modeling alcoholic liver disease in a human Liver-Chip. bioRxiv 2020.07.14.203166 (2020) doi:10.1101/2020.07.14.203166. 
31. Jang, K.-J. et al. Reproducing human and cross-species drug toxicities using a Liver-Chip. Science

Translational Medicine 11, eaax5516 (2019).

32. Sheyn, D. et al. Bone-chip system to monitor osteogenic differentiation using optical imaging.

Microfluid Nanofluid 23, 99 (2019).

33. Barrile, R. et al. Organ-on-Chip Recapitulates Thrombosis Induced by an anti-CD154 Monoclonal Antibody: Translational Potential of Advanced Microengineered Systems. Clinical Pharmacology \& Therapeutics 104, 1240-1248 (2018).

34. Tan, J. L., Liu, W., Nelson, C. M., Raghavan, S. \& Chen, C. S. Simple approach to micropattern cells on common culture substrates by tuning substrate wettability. Tissue Eng 10, 865-872 (2004).

35. Amatngalim, G. D. et al. Aberrant epithelial differentiation by cigarette smoke dysregulates respiratory host defence. European Respiratory Journal 51, 1701009 (2018).

36. Xia, G., Tawhai, M. H., Hoffman, E. A. \& Lin, C.-L. Airway Wall Stiffening Increases Peak Wall Shear Stress: A Fluid-structure Interaction Study in Rigid and Compliant Airways. Ann Biomed Eng 38, 1836-1853 (2010).

37. Schrumpf, J. A., Ninaber, D. K., van der Does, A. M. \& Hiemstra, P. S. TGF- $\beta 1$ Impairs Vitamin DInduced and Constitutive Airway Epithelial Host Defense Mechanisms. J Innate Immun 12, 74-89 (2020).

38. Copland, I. B., Reynaud, D., Pace-Asciak, C. \& Post, M. Mechanotransduction of stretch-induced prostanoid release by fetal lung epithelial cells. American Journal of Physiology-Lung Cellular and Molecular Physiology 291, L487-L495 (2006).

39. Thomas, R. A. et al. Mechanical stretch has contrasting effects on mediator release from bronchial epithelial cells, with a rho-kinase-dependent component to the mechanotransduction pathway. Respir Med 100, 1588-1597 (2006). 
40. Vlahakis, N. E., Schroeder, M. A., Limper, A. H. \& Hubmayr, R. D. Stretch induces cytokine release by alveolar epithelial cells in vitro. American Journal of Physiology-Lung Cellular and Molecular Physiology 277, L167-L173 (1999).

41. Oudin, S. \& Pugin, J. Role of MAP kinase activation in interleukin-8 production by human BEAS2B bronchial epithelial cells submitted to cyclic stretch. Am J Respir Cell Mol Biol 27, 107-114 (2002).

42. Cruz, F. F., Rocco, P. R. M. \& Pelosi, P. Role of the extracellular matrix in the genesis of ventilator-induced lung injury. Med Klin Intensivmed Notfmed 113, 2-6 (2018).

43. Wang, J. et al. Transient receptor potential canonical 1 channel mediates the mechanical stress-induced epithelial-mesenchymal transition of human bronchial epithelial (16HBE) cells. Int J Mol Med 46, 320-330 (2020).

44. Trieu, D., Waddell, T. K. \& McGuigan, A. P. A microfluidic device to apply shear stresses to polarizing ciliated airway epithelium using air flow. Biomicrofluidics 8, 064104 (2014).

45. Karra, N., Swindle, E. J. \& Morgan, H. Drug delivery for traditional and emerging airway models. Organs-on-a-Chip 1, 100002 (2019).

46. Weibel, E. R. Morphometry of the Human Lung. (Springer-Verlag, 1963). doi:10.1007/978-3-64287553-3.

47. Patton, J. S. \& Byron, P. R. Inhaling medicines: delivering drugs to the body through the lungs. Nat Rev Drug Discov 6, 67-74 (2007).

48. Rayner, R. E., Makena, P., Prasad, G. L. \& Cormet-Boyaka, E. Optimization of Normal Human Bronchial Epithelial (NHBE) Cell 3D Cultures for in vitro Lung Model Studies. Scientific Reports 9, 500 (2019).

49. Nawroth, J. C., van der Does, A. M., Ryan (Firth), A. \& Kanso, E. Multiscale mechanics of mucociliary clearance in the lung. Philosophical Transactions of the Royal Society B: Biological Sciences 375, 20190160 (2020). 
50. Mercer, R. R., Russell, M. L., Roggli, V. L. \& Crapo, J. D. Cell number and distribution in human and rat airways. Am J Respir Cell Mol Biol 10, 613-624 (1994).

51. Schiller, H. B. et al. The Human Lung Cell Atlas: A High-Resolution Reference Map of the Human Lung in Health and Disease. Am J Respir Cell Mol Biol 61, 31-41 (2019).

52. Yang, J. et al. Smoking-Dependent Distal-to-Proximal Repatterning of the Adult Human Small Airway Epithelium. Am. J. Respir. Crit. Care Med. 196, 340-352 (2017).

53. Tadokoro, T., Gao, X., Hong, C. C., Hotten, D. \& Hogan, B. L. M. BMP signaling and cellular dynamics during regeneration of airway epithelium from basal progenitors. Development 143, 764773 (2016).

54. Xu, J., Liu, M. \& Post, M. Differential regulation of extracellular matrix molecules by mechanical strain of fetal lung cells. Am J Physiol 276, L728-735 (1999).

55. Hackett, N. R. et al. The Human Airway Epithelial Basal Cell Transcriptome. PLoS ONE 6, e18378 (2011).

56. Jeffery, P. K., Gaillard, D. \& Moret, S. Human airway secretory cells during development and in mature airway epithelium. Eur Respir J 5, 93-104 (1992).

57. Chapman, K. E. et al. Cyclic mechanical strain increases reactive oxygen species production in pulmonary epithelial cells. Am J Physiol Lung Cell Mol Physiol 289, L834-841 (2005).

58. Tschumperlin, D. J., Oswari, J. \& Margulies, A. S. Deformation-induced injury of alveolar epithelial cells. Effect of frequency, duration, and amplitude. Am J Respir Crit Care Med 162, 357-362 (2000).

59. Brown, K. R. S., England, K. M., Goss, K. L., Snyder, J. M. \& Acarregui, M. J. VEGF induces airway epithelial cell proliferation in human fetal lung in vitro. American Journal of Physiology-Lung Cellular and Molecular Physiology 281, L1001-L1010 (2001). 
60. Asano, S. et al. Matrix stiffness regulates migration of human lung fibroblasts. Physiol Rep 5,

(2017).

61. Huang, X. et al. Matrix Stiffness-Induced Myofibroblast Differentiation Is Mediated by Intrinsic Mechanotransduction. Am J Respir Cell Mol Biol 47, 340-348 (2012).

62. Peters-Hall, J. R. et al. Long-term culture and cloning of primary human bronchial basal cells that maintain multipotent differentiation capacity and CFTR channel function. Am J Physiol Lung Cell Mol Physiol 315, L313-L327 (2018).

63. Abramoff, M., Magelhaes, P. \& Ram, S. Image processing with ImageJ. Biophotonics Int 11, 3642 (2004).

64. Escobar-Chávez, J. J. et al. Applications of thermo-reversible pluronic F-127 gels in pharmaceutical formulations. J Pharm Pharm Sci 9, 339-358 (2006).

65. ZUIDERVELD, K. Contrast Limited Adaptive Histogram Equalization. Graphics Gems IV, 474-485 (1994).

66. Schindelin, J. et al. Fiji: an open-source platform for biological-image analysis. Nat Meth 9, 676682 (2012).

67. Edelstein, A., Amodaj, N., Hoover, K., Vale, R. \& Stuurman, N. Computer control of microscopes using $\mu$ Manager. Curr Protoc Mol Biol Chapter 14, Unit14.20 (2010).

68. Jaqaman, K. et al. Robust single-particle tracking in live-cell time-lapse sequences. Nat Methods 5, 695-702 (2008). 
A

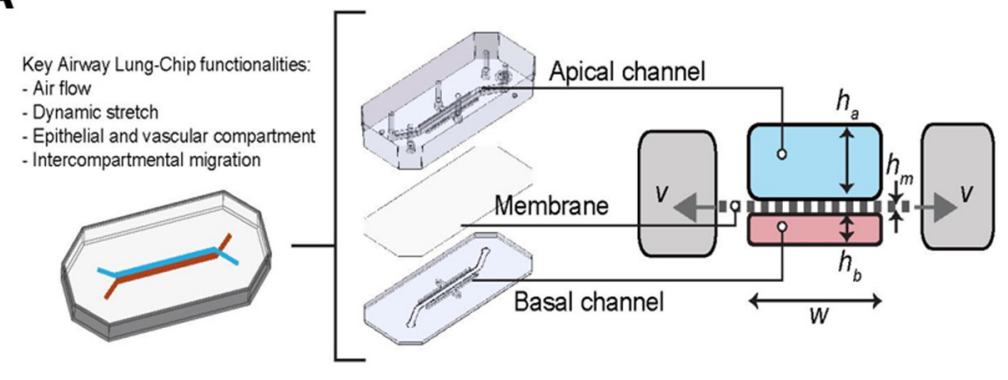

C

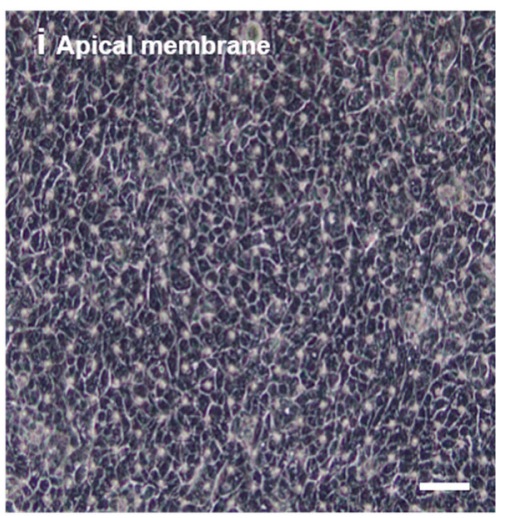

D

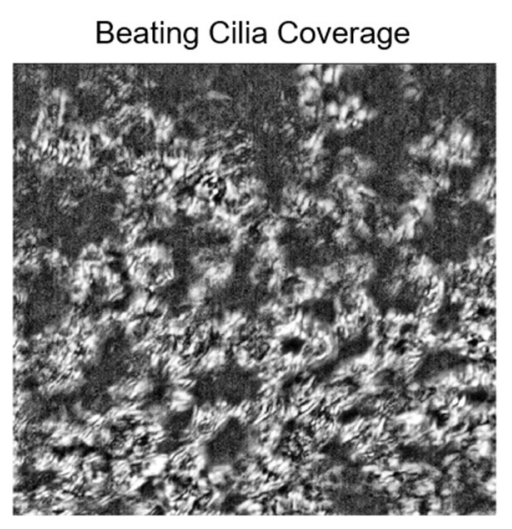

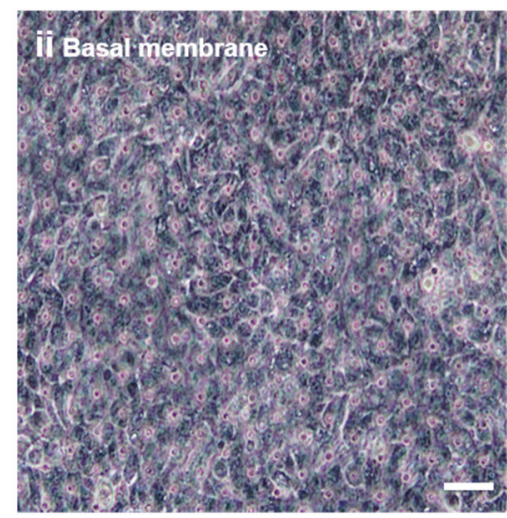

$E$
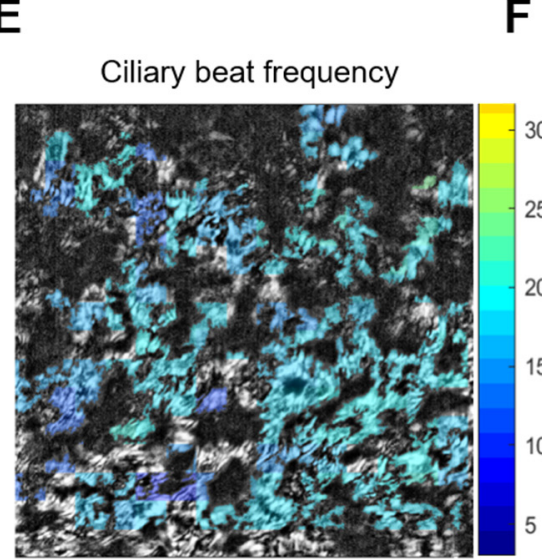

$\mathbf{F}$
B
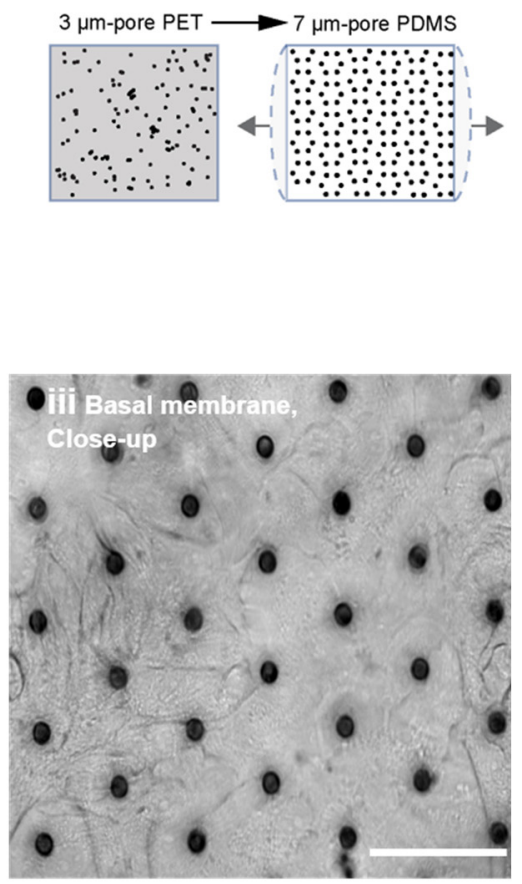
Mucus clearance

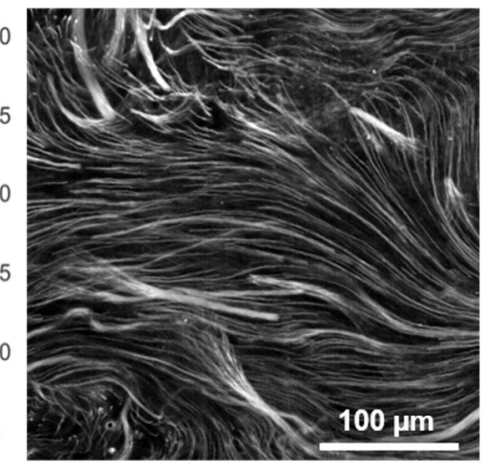

Figure S1: Benefits and challenges of the Chip-S1 design for pHBEC studies.

A, The Chip-S1 supports key functionalities for in vitro airway studies through the perfusable dualcompartment design and porous vacuum $(v)$-actuatable membrane. $w$, channel width $=1000 \mu \mathrm{m} ; h_{a}$, apical channel height $=1000 \mu \mathrm{m} ; h_{b}$, basal channel height $=200 \mu \mathrm{m} ; h_{m}$, membrane thickness $=25 \mu \mathrm{m}$. B, The major upgrade from previous Airway Lung-Chip studies is the switch from a rigid, track-etched, and translucent PET membrane to a flexible PDMS membrane with exact $7 \mu \mathrm{m}$ diameter pore size and optical transparency. C, Phase contrast microscopy of left: the apical side of membrane showing the pHBEC at $\mathrm{ALI}$ and middle/right: the basal side of membrane showing a confluent layer of pHBEC that have migrated to the bottom channel. Scalebars: $50 \mu \mathrm{m}$. D, Motion detection of ciliary beat (white regions). E, Analysis of high-speed phase contrast videos (top) enabled measurement of ciliary beat frequency (CBF). F, Video recordings of fluorescent bead trajectories enabled measurement of mucus clearance. 
A Hydrogel in apical channel

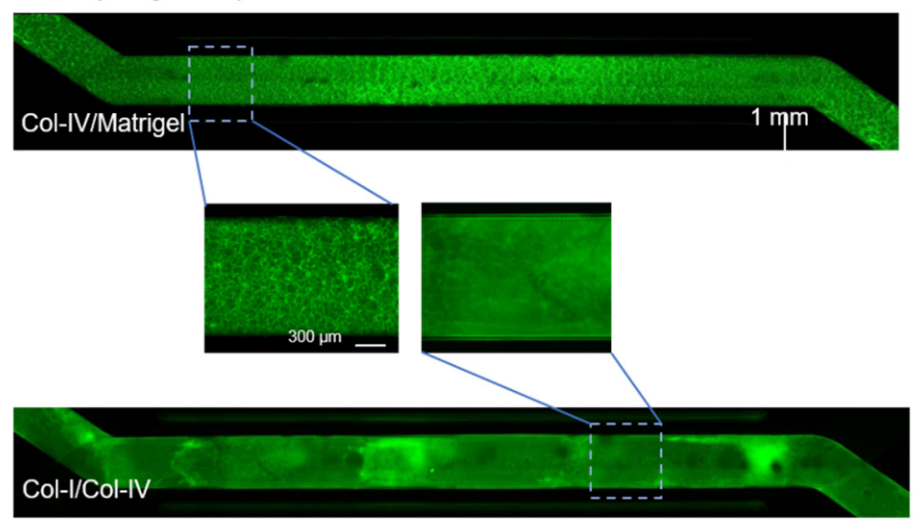

C

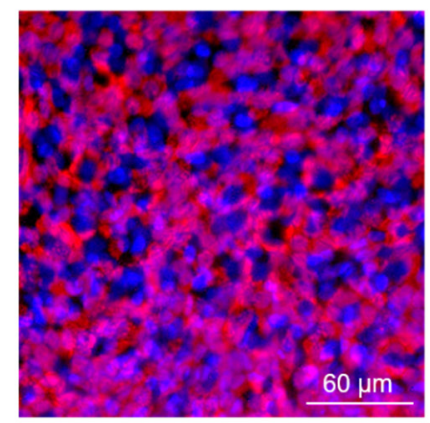

ACTUB DAPI

D

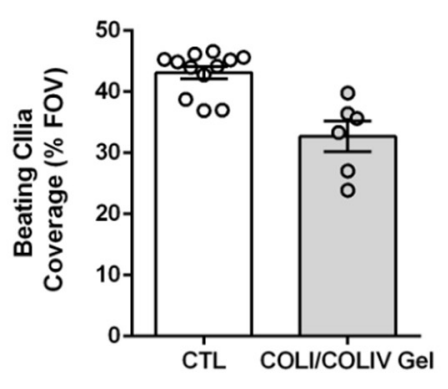

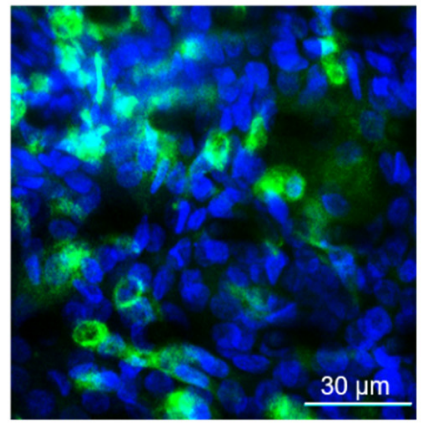

Muc5AC DAPI

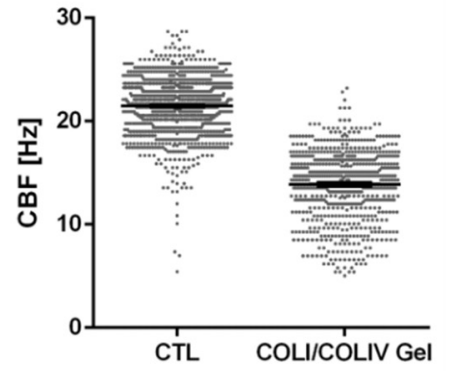

B

Gel Geometry
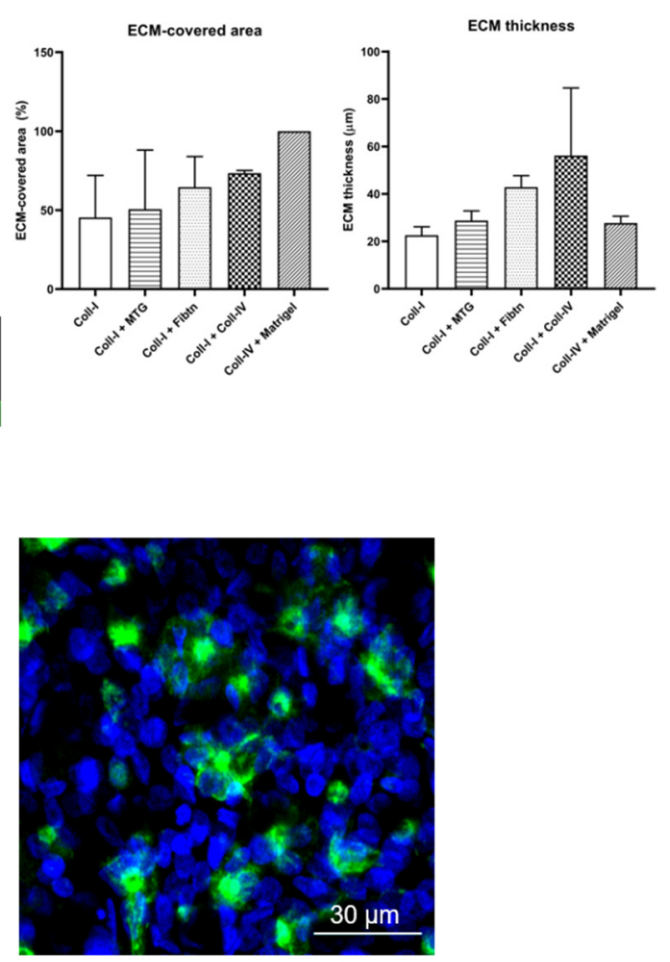

SCGB1A1 DAPI

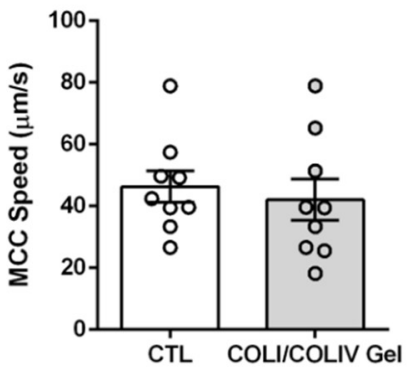

Figure S2: Hydrogel barrier in apical channel prevents pHBEC migration and supports pHBEC differentiation at ALI.

A, Representative images of hydrogels (green) consisting of collagen I/Matrigel or collagen I/collagen IV lining the entire membrane in the apical channel. B, Compared to other gel formulations, the gel compositions in (A) achieved superior percentage of membrane coverage (left) combined with robust gel thicknesses above $20 \mu \mathrm{m}$ (right). C, Immunofluorescent (IF) staining of hydrogel chips at day 14 ALI shows presence of 3 major differentiated airway epithelial cell types: acetylated $\alpha$-tubulin (ciliated cells), Muc5AC (goblet cells), SCGB1A1 (club cells). Nuclei are stained with DAPI. D, Comparison of ciliary function in hydrogel chips and BSA-coated control chips with no or mild invasion. Left: Relative coverage of surface with beating cilia. Each data point represents 1 field of view. Column and error bars shown represent 
mean +/- SEM. Center: Ciliary beat frequency (CBF). Each data point represents approximately 1 ciliated cell. Lines are mean +/- SEM. Right: Measurement of mucus clearance speed. Each data point represents a 1 field of view. Column and error bars shown represent mean +/- SEM. Data in are from $\mathrm{N}=1$ donor with 1 chip at day $25 \mathrm{ALI}$ (Gel) and 2 chips (CTL) at day $20 \mathrm{ALI}$.

A
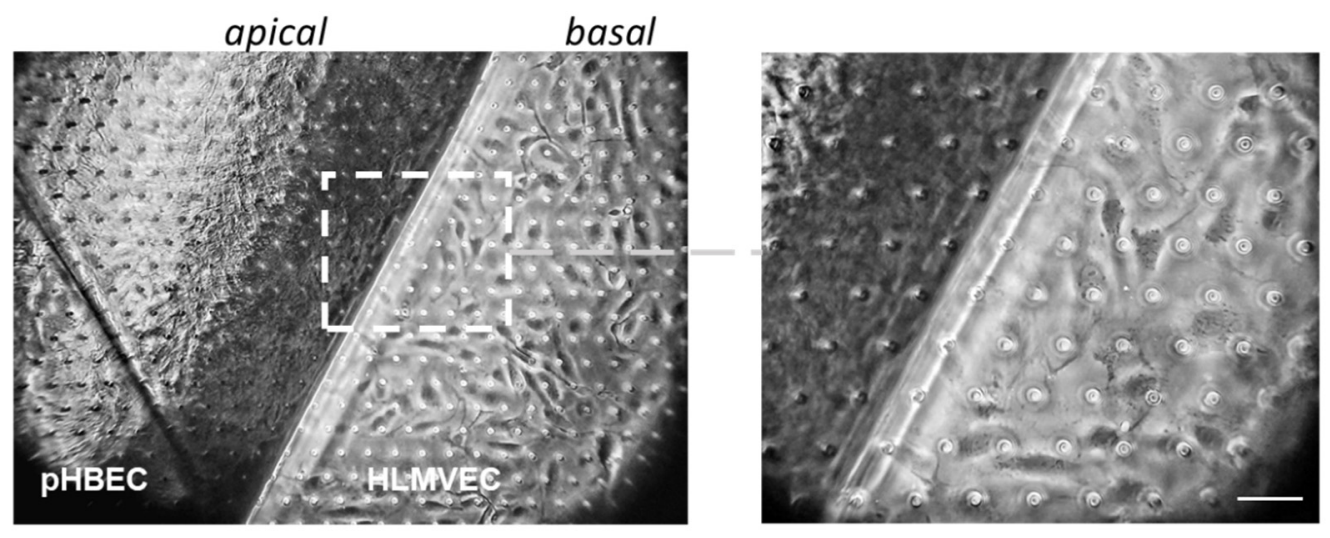

B
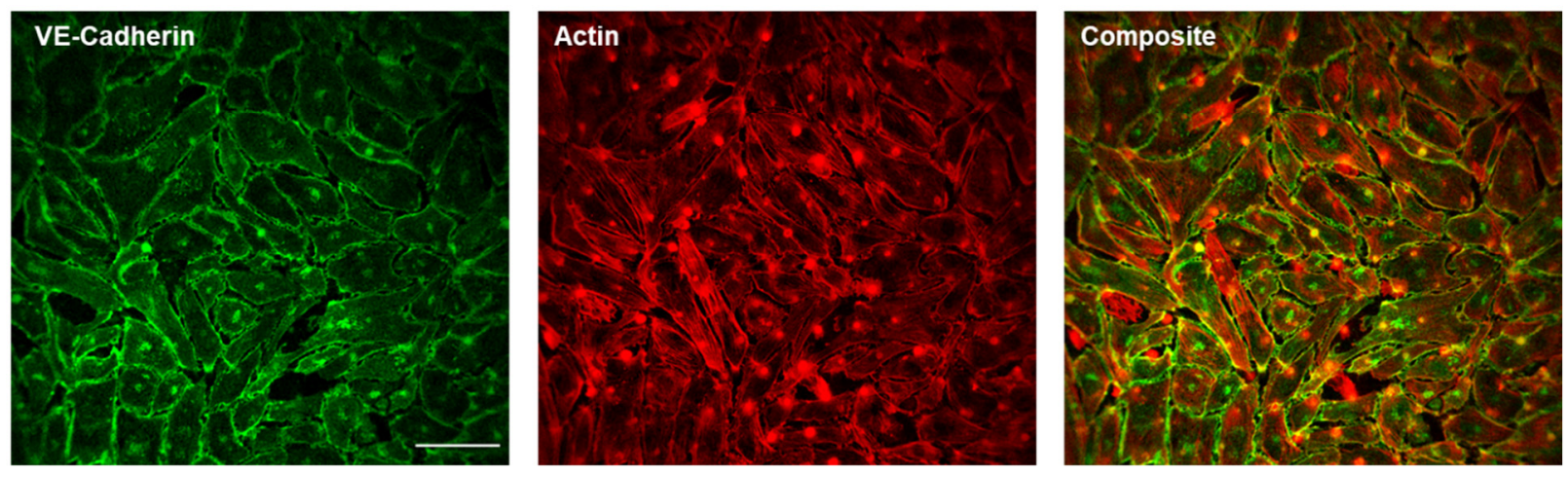

Figure S3: Endothelial co-culture on invasion-free Airway Lung-Chips.

A, Left: Phase contrast image of co-culture of pHBEC in apical channel and HLMVEC in basal channel, on opposing sides of the PDMS membrane (shown here is junction where the channels diverge), $3 \mathrm{~h}$ after seeding of the endothelial cells at day 12 of ALI. Right: inset showing typical endothelial morphology and successful adhesion to the fibronectin-coated PDMS membrane. Scale bar: $50 \mu \mathrm{m}$. B, IF stain showing that HLMVEC lining the basal channel membrane express the endothelial-specific cell junction marker vascular endothelial (VE)-cadherin at day 14 of ALI, i.e., 48h post-seeding of the endothelial cells. Note that the regularly spaced, circular structures are the auto-fluorescent membrane pores. Scale bar: $100 \mu \mathrm{m}$. Data from one donor, 2 chips. 